\title{
Supplier Diversity before the Time of Cholera
}

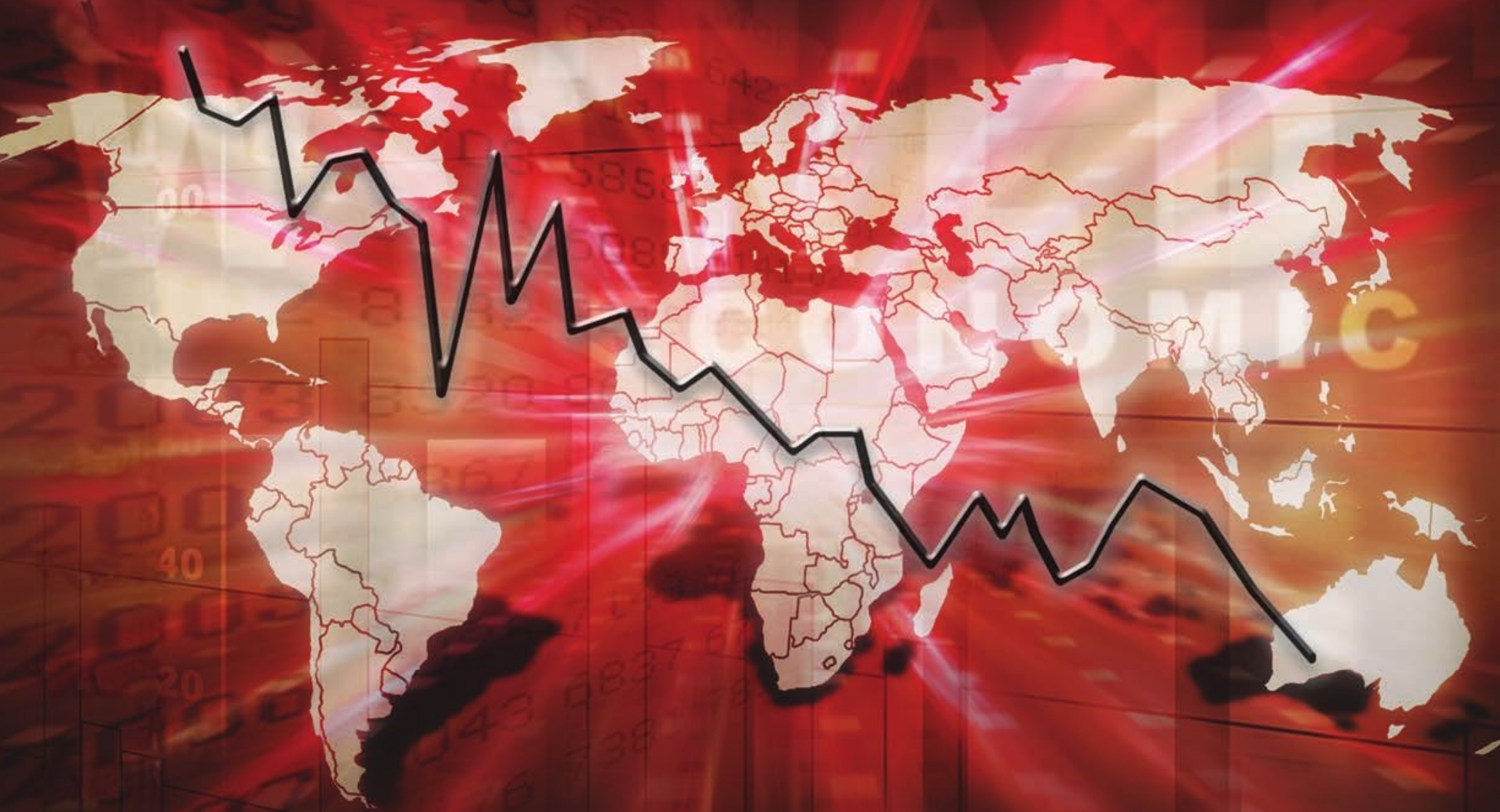

José Alcalde, University of Alicante Matthias Dahm, University of Leicester

Working Paper No. 2007 April 2020 


\title{
Supplier Diversity before the Time of Cholera*
}

\author{
José Alcalde $\quad$ Matthias Dahm
}

April 9, 2020

*The authors appreciate the helpful suggestions of Fabrizio Adriani, Subir Bose and Guiseppe de Feo, as well as the participants at the fifth annual conference "Contests: Theory and Evidence," University of East Anglia, UK. This research was conducted while José Alcalde was visiting the University of Nottingham, whose generous hospitality is gratefully acknowledged. This work is partially supported by the Spanish Ministerio de Economía y Competitividad, project ECO2016-77200-P.

${ }^{\dagger}$ Corresponding Author. IUDESP, University of Alicante. ORCID: orcid.org/0000-0001-7491-4524. jose.alcalde@ua.es

+Department of Economics, University of Leicester. ORCID: orcid.org/0000-0002-3146-6802. matthias.dahm@leicester.ac.uk 


\begin{abstract}
Procurement programs often aim to rely on a diverse pool of suppliers, besides achieving cost effectiveness. We propose complementing a share auction for dual sourcing with affirmative action to create an endogenous set-aside for a high-cost supplier. In our model more intensive affirmative action strengthens the targeted provider. This has the potential to level the playing field, inducing more competitive procurement overall. Our main result provides a condition under which the endogenous set-aside not only guarantees a very substantial share for the high-cost supplier, but also reduces the buyer's provision cost compared to a first-price auction. This result is robust to variations of our benchmark model, including the assumptions specifying what providers know about each other, and how affirmative action programs are implemented. We also illustrate how our approach can help to reduce the severity and likelihood of health product shortages, such as those that occurred during the recent COVID-19 outbreak.
\end{abstract}

Keywords: Affirmative Action, Bidding Credits, Bidding Preferences, Set-Asides, Dual Sourcing. Journal of Economic Literature Classification Numbers: D44, D47, H57, J15. 
And ... after the crisis, I believe, ..., we also have to identify many strategic products that we have to insist will be manufactured in Germany, or ... Europe, in the future, ... This will also apply to medicines and many other things. ... [This] will make some things more expensive, but that's the right thing to do.

Olaf Scholz, Federal Minister of Finance and Vice Chancellor of Germany $\left.\right|^{1}$

\section{Introduction}

Procurement programs often aim to rely on a diverse pool of suppliers. There are at least two reasons for this. First, supplier diversity is a resilience measure that makes supply chains more robust to shocks. With a dual sourcing strategy, for instance, a supply contract is sourced from more than one provider. This avoids to be locked in with a provider in the event that he cannot fulfil his obligations. ${ }^{2}$ Following the recent outbreak of COVID-19 many firms around the world in many different sectors experienced severe disruptions to their supply chains. In response to these challenges many of these firms will make more use of dual sourcing. ${ }^{3}$ Second, supplier diversity is also an end in itself. Government procurement programs for example

\footnotetext{
${ }^{1}$ On 05/04/2020 on the ARD talk show 'Anne Will' discussing the COVID-19 outbreak, see https: //www.daserste.de/information/talk/anne-will/videos/index.html, minutes 15:50-16:27, accessed on 05/04/2020. Translation by the authors. Olaf Scholz said: "Und für nach der Krise, glaube ich, ..., dass wir auch viele strategische Produkte identifizieren müssen, bei denen wir darauf bestehen müssen, dass sie in Zukunft auch in Deutschland, oder ... in Europa hergestellt werden, ... Das wird auch für Arzneimittel gelten und für viele andere Dinge. ... [dies] wird dazu führen, dass manches teurer wird, ..., aber das ist dann auch richtig so."

${ }^{2}$ In Gabriel García Márquez's novel, 'Love in the Time of Cholera,' love is compared to a disease, such as cholera. In contrast, supplier diversity is a precautionary resilience measure implemented before a disease, such as COVID-19, materialises. Following the Olaf Scholz's intervention in the talk show 'Anne Will' (see footnote 1), a prominent German economics professor recommended supply chain diversification, rather than renationalisation of supply chains, as an important lesson from the COVID-19 outbreak.

${ }^{3}$ For instance, the European Medicines Agency reports that "While the purpose of the group is to address disruptions of the supply of medicines in the EU through a coordinated approach, it is important to highlight that it is the responsibility of pharmaceutical companies to ensure the continuity of supply of their medicines. This includes for instance that manufacturers put in place appropriate resilience measures such as the increase of stocks or dual sourcing of products and materials." (See the article "Addressing the potential impact of novel coronavirus disease (COVID-19) on medicines supply in the EU)." Similarly, the Executive Summary of the SEMI Industry Survey on the Impact of COVID 19, reports that "With substantial disruptions in supply chains of materials, equipment and services, companies are accelerating plans in place for dual-sourcing. Longer term we will likely see dual-sourcing as a key theme in business expansion and contingency plans." The article "Covid-19: The impact on supply chains," by Fidelity International, reports that "To reduce this risk, dual sourcing is used in most industries, including automotives and tech hardware, but it's not a perfect solution. It can take months to ramp up production from alternative suppliers. Apple is a company with a dual sourcing policy that appears robust, but it is facing production delays at its Shenzhen supplier; alternate production sites in northern China and India do not have the same scale." All pages accessed on March 31, 2020.
} 
favour firms with specific characteristics to further certain social and political purposes. For instance, in the U.S., the Small Business Act and other programs at the level of states and localities specify geographical preferences for American-made (or state-made) commodities and non-geographical preferences, including for small, minority, disadvantaged, veteran-owned or women-owned businesses as well as for environmentally friendly commodities. ${ }^{4}$

Common wisdom, however, holds that supplier diversity is in conflict with the aim of allocating supply contracts in a cost effective way. For instance Baldwin and Tomiura (2020, p. 69) argue that dual sourcing helps mitigating the consequences of COVID-19 but describe the trade-off as follows: "Redundant dual sourcing from multiple countries alleviates the problem of excess dependence on China, though with additional costs.' 5 In the context of affirmative action, preference programs are also thought to be costly for society and this provides an important argument for opponents of affirmative action. 6

In this paper we explore the trade-off between cost effective procurement and supplier diversity. Our model complements a share auction for dual sourcing with affirmative action to create endogenous set-asides.7 Our main result is to show that this has the potential to reconcile the conflicting aims of cost effective procurement and supplier diversity.

As reported by Carpineti et al. (2006), procurement auctions are usually organized as firstprice auctions (FPA hereafter). According to these authors, many organizations do not deter-

${ }^{4}$ The U.S. Small Business Administration aims to allocate at least 23 percent of all federal contracting dollars to small businesses, see www.sba.gov/federal-contracting/ contracting-assistance-programs accessed on 22/07/2019. Nakabayashi (2013) reports that Japan has a similar program. See Qiao et al. (2009) for a survey of forms of preferences in public procurement in the U.S. and other countries, and Holzer and Neumark (2000) for an economic assessment of affirmative action programs, including procurement. Mummalaneni (2019) reports that companies in the private sector, including Chevron, Coca-Cola, Microsoft, and MillerCoors, have "Tier II" procurement programs favouring minority vendors.

${ }^{5}$ This is in line with the view that dual sourcing "almost certainly includes one supplier that is not at least cost" (Albano et al., 2006, p. 110) and hence the "disadvantage is that the price is in general higher than with single sourcing" (Engel et al., 2006, p. 330).

${ }^{6}$ Based on their findings Qiao et al. (2009, p. 396) describe the trade-off as follows: "In summary, the most interesting and important finding regarding the impact of a preference program is that many people believe that preference programs violate the free-competition principles and these programs cause government to pay a higher price ..." Ayres and Cramton (1996) for example report that various California ballot initiatives tried to end state-sponsored affirmative action because of the belief that eliminating affirmative action could help to solve budget problems. Jehiel and Lamy (2015) mention that the World Trade Organization (WTO) and the European Commission rule out discrimination because they fear that it leads to higher prices. Marion (2009) finds that the elimination of the consideration of race or gender in awarding state-funded highway construction contracts in California saved an estimated \$64 million in the 1998-1999 period.

${ }^{7}$ When government procurement programs aim to achieve supplier diversity as a resilience measure that makes supply chains more robust to shocks, they are following an industrial policy, rather than an affirmative action policy in a strict sense. For conciseness, however, we use the term affirmative action. Throughout the paper we will measure supplier diversity by the smaller procurement share of the two providers. In Section 5 we will show that supplier diversity is a better precautionary resilience measure the larger this share. 
mine the reserve price in the way the optimal mechanism would require. A similar benchmark is used in Ewerhart and Fieseler (2003) and Alcalde and Dahm (2013, 2019). We derive our main result by comparing the benchmark of the FPA to our share auction complemented by affirmative action. Using the language in Jehiel and Lamy (2019) we take a positive perspective that seeks to contribute to the policy debate on affirmative action, rather than a normative perspective that investigates the optimal auction mechanism (Myerson, 1981).

Under the FPA format the buyer specifies the kind of supply contract offered and the budget constraint (or reserve price). The potential providers propose prices at which they are willing to supply the contract and the supplier submitting the lowest price is chosen. Frequently, procurement programs implement preferential treatment in the form of set-aside programs that reserve some contracts for certain categories of bidders ${ }^{8}$ In a similar way, following the COVID-19 outbreak, some countries plan to incentivise a domestic provider to supply certain goods by giving a guarantee to buy the supplier's total production.9 9 We proceed in a similar way as the FPA but endogenize set-asides by assigning each provider a share that depends on the prices submitted. To do so we complement the Contested Procurement Auction (CPA hereafter) introduced in Alcalde and Dahm (2013) with affirmative action. The CPA is a share auction for dual sourcing that allocates shares of the supply contract depending on the prices of suppliers. To describe more precisely how these shares are computed, define a supplier's bid to be the difference between the budget constraint and the price of this supplier. The CPA assigns shares depending on the relative difference of the bids of suppliers, as a percentage of the largest bid (submitted by the supplier proposing the lowest price).

To fix ideas we consider a procurement problem with a local and a foreign supplier under complete information 10 Since we are interested in affirmative action considerations, we assume that the foreign provider is more efficient than his local rival ${ }^{11}$. We show that an affirmative action program targeting the local supplier has the potential to make procurement more competitive. This is because it makes the local provider stronger, inducing him to set a lower price than he otherwise would ${ }^{12}$ The foreign supplier in turn responds to this by setting a more competitive price too. Our first result is a characterization of equilibrium for any intensity of affirmative action. As this intensity increases, equilibrium supplier diversity, that is, the local providers' equilibrium market share rises until the playing field is completely levelled. If

\footnotetext{
${ }^{8}$ For instance the U.S. Small Business Administration allocates procurement contracts using set-asides, see www.sba.gov/federal-contracting/contracting-assistance-programs accessed on 22/07/2019.

${ }^{9}$ For instance, Olaf Scholz mentions his intention to adopt such a policy in the talk show 'Anne Will', see footnote 1 .

${ }^{10}$ The assumption of complete information is considered appropriate for situations in which sellers know each other well (Moldovanu and Sela, 2003). The case of construction contracting (Bernheim and Whinston, 1986) and settings with stable technology (Anton and Yao, 1992, p. 691) are examples. In Subsection 4.2 we modify the procurement auction to relax this assumption.

${ }^{11}$ Besides affirmative action considerations, there could be a National Security concern to make sure that certain goods are produced nationally, as for many health products during the COVID-19 outbreak.

${ }^{12}$ We will see that the local provider becomes stronger in the sense that for low intensities of affirmative action he acts as if his costs were lower than they really are.
} 
the intensity increases further, this might even result in an imbalance in favour of the domestic supplier. Similarly, any increase in the intensity of affirmative action raises the profits of the local provider and reduces those of the foreign supplier. This sensitivity of the consequences of affirmative action under the CPA contrasts with the FPA. This is so, since under the FPA equilibrium supplier diversity and the local provider's profits are zero, unless the intensity level of the affirmative action program is sufficient to level the playing field completely. 13

Our main result provides a condition under which complementing the CPA with affirmative action reconciles the conflicting aims of supplier diversity and cost effectiveness. To do so the buyer has to choose the intensity of affirmative action in such a way that, at the equilibrium, both providers select the same price. This levels the playing field completely and supplier diversity is maximal, as the equilibrium market shares of both providers are equal. Moreover, provision costs are lower than those arising from a standard first-price auction for sole sourcing, so that the supply contract is allocated in a cost effective way. The condition under which this result holds requires the cost difference of providers to be sufficiently large. The possibility to use affirmative action benefits the buyer, because the trade-off between supplier diversity and cost effectiveness disappears for a smaller cost difference than in our earlier paper Alcalde and Dahm (2013).

The benchmark model can be extended in different ways. First, we consider alternative affirmative action programs. Two prominent ways to introduce affirmative action into procurement auctions are subsidies and biases affecting the award rule. An example for the latter are bidding preferences (or bidding credits) in which the prices of targeted firms are lowered by a specified percentage amount. ${ }^{14}$ Our benchmark model considers affirmative action in form of a subsidy. Our first extension provides an equivalence result between the equilibrium of an affirmative action program affecting the award rule and the equilibrium of a program providing a subsidy. We also discuss limitations of this equivalence that come from the fact that -unlike in standard contest and bidding games- in our setting the providers' strategy spaces are bounded. Second, our benchmark model assumes that providers have complete information about their rival's costs. Since there are many situations in which this assumption is not appropriate, we discuss a version of our share auction that allows to consider providers with private information about their costs. Lastly, we also explain how our benchmark model might be extended to

\footnotetext{
${ }_{13}^{13}$ Ayres and Cramton (1996, p. 7) give a related example of a traditional English (or open ascending) auction among two strong and two weak bidders for two licenses. Without bidding credit and with a $25 \%$ bidding credit the two strong bidders obtain licenses but with a 50\% bidding credit one weak and one strong bidder obtain a license.

${ }^{14}$ Both subsidies and bidding credits are employed in the U.S. by the Federal Communications Commission in spectrum auctions and under the Buy America Act, see Athey et al. (2013) and Loertscher and Marx (2017). Subsidies are used in California state highway procurement (Athey et al., 2013) and twenty-five U.S. states provide bid preferences or set-asides for in-state bidders or products (Loertscher and Marx , 2017). While the level of a bidding credit to be applied is usually known, this is not true in the Virginia public procurement market, where suppliers know whether they are eligible to receive a bid credit, but they do not know the bid credit level that will be applied (Mummalaneni, 2019). There is also the "right of first refusal" that gives a favoured bidder the opportunity to win the supply contract by matching the best bid of the competing bidders, see Lee (2008) for an analysis.
} 
multiple sourcing.

As mentioned before, this paper builds on our previous work. Alcalde and Dahm (2013) consider a family of assignment rules to allocate procurement shares that differ in the sensitivity of a supplier's share with regard to his price. The CPA in the present paper constitutes the case of unit elasticity of this family. The main result in Alcalde and Dahm (2013) says that for any values of the providers' costs one can always find a level of sensitivity such that procurement costs are lower than with a standard first price auction for sole sourcing. The present paper departs from the case of unit elasticity in our earlier paper by introducing affirmative action programs, rather than by considering different levels of sensitivity to prices. This allows not only to say that an optimal choice exists -as in our earlier paper- but also to describe it in a simple closed-form. Alcalde and Dahm (2019) consider more than two providers and use the additional suppliers to endogenize the reserve price but do not introduce affirmative action. While attracting further suppliers also has the potential to lead to very competitive procurement, total costs depend on the costs of the providers that participate. Hence, from a practical point of view the optimal intensity of affirmative action derived in the present paper is easier to target and to control than the design parameters considered in our earlier papers.

In a recent paper Jehiel and Lamy (2019) also take the auction format as given and investigate the effects of set-asides on procurement expenditure. In their model there is an incumbent who participates for sure and a set of potential entrants whose participation is endogenous. Jehiel and Lamy discover an exclusion principle. It is always beneficial to (completely) exclude the incumbent. The intuition for this is that exclusion stimulates participation and thereby competition. In contrast, in our model the set of participants is fixed and the share auction excludes the foreign supplier partially from the supply contract. Affirmative action makes the local provider stronger and induces the foreign supplier to set a lower price than he otherwise would. We establish a partial exclusion principle. We provide a condition under which it is beneficial to use affirmative action in our share auction to set aside half of the supply contract.

By combining a share auction (akin to a contest success function) with a winner-pay (rather than an all-pay) payment rule, the present paper contributes to bridge the literatures on auctions and contests. The introduction of affirmative action in our CPA levels the playing field and has the potential to strengthen competition between suppliers. This parallels findings in the literature on auctions for an indivisible object with asymmetric bidders, where revenue maximization requires discrimination in the sense that sometimes the item is not awarded to the bidder whose value estimate is the highest (Myerson, 1981; Maskin and Riley, 2000) 15 In procurement auctions, affirmative action in favour of a high-cost provider in form of subsidies (Ewerhart and Fieseler, 2003; Rothkopf et al., 2003) and in form of bidding preferences (Ayres and Cramton,

\footnotetext{
15 Jehiel and Lamy (2015) analyse optimal discrimination in auctions when entry is endogenous. The assignment of shares also connects our paper to the literature on share and split-award auctions that explores conditions under which sole sourcing is more advantageous than a split-award (Wilson, 1979; Bernheim and Whinston, 1986; Anton and Yao, 1989, 1992; Perry and Sákovics, 2003; Bag and Li, 2014). A major difference is that our allocation rule for procurement shares imposes a particular structure on the trade-off a supplier faces when deciding on his price.
} 
1996; McAfee and McMillan, 1989; Hubbard and Paarsch, 2009) can foster competition. ${ }^{16}$ Similarly, in contests biases in the assignment rule of the prize that resemble bidding preferences can increase total effort (Franke, 2012; Franke et al., 2013, 2014, 2018), 17

This paper is organized as follows. The next section introduces the procurement problem, the CPA assignment rule and affirmative action in form of a subsidy. We conduct our strategic analysis in Section 3 . Section 4 discusses the aforementioned extensions of this setting. Section 5 discusses how our approach would help to mitigate some of the provision problems that appeared during the COVID 19 crisis when, in line with Katz and Singer (2007), Health is considered a National Security concern. The last section contains concluding remarks. All proofs are relegated to the Appendix.

\section{The Affirmative Action Procurement Problem}

\subsection{The Procurement Problem}

We consider a buyer who wishes to buy a given amount of a perfectly divisible good. The size of this supply contract is normalized to one. The buyer's budget (or reserve price) is denoted by $b$ and represents the maximum expenditure possible. There are two potential providers (or suppliers): the local provider, denoted by $\ell$, and a foreign supplier, denoted by $\mathfrak{f}$. The suppliers' technologies exhibit constants returns to scale, so that average and marginal costs are constant. Let $c_{i}$ denote the marginal cost of provider $i \in\{\ell, \mathfrak{f}\}$. We will refer to the tuple $\left(b, c_{\ell}, c_{\mathfrak{f}}\right)$ as a procurement problem.

Since we are interested in affirmative action programs, we suppose that the local provider is less efficient than the foreign supplier. For operational purposes it is also assumed that the buyers' budget is not too restrictive. More precisely, we assume that $0 \leq c_{\mathfrak{f}}<c_{\ell}<b$. We also assume that the suppliers are perfectly informed about the costs of both providers and the buyer's budget constraint, while the buyer does not know the providers' cost. ${ }^{18}$

The buyer organises a (simultaneous) bidding game among the suppliers. To fix ideas we present this game first without yet incorporating affirmative action considerations. Each poten-

\footnotetext{
${ }^{16}$ The empirical literature obtains mixed results. This is consistent with our model, as the level playing effect of affirmative action depends on its intensity. Analysing small business set-asides, Denes (1997) finds no significant cost savings in all but one instance. Support for strengthened competition comes from radio spectrum auctions (Ayres and Cramton, 1996), experimental evidence (Corns and Schotter, 1999), timber auctions (Brannman and Froeb, 2000), snow removal contracts in Montreal (Flambard and Perrigne, 2006) as well as Japanese and Virginia public procurement markets (Nakabayashi, 2013; Mummalaneni, 2019), while studies of road construction contracts (Marion, 2007, 2009; Krasnokutskaya and Seim, 2011) and of timber auctions (Athey et al., 2013) find that procurement costs are increased.

${ }^{1 /}$ Chowdhury et al. (2019) review the theoretical and empirical literature on level the playing field policies and affirmative action in contests. We clarify the relationship between out model and a standard contest setting in Appendix A.5. The serial contest in Alcalde and Dahm (2007) combines a contest success function that is closely related to the way in which the CPA assigns shares with an all-pay payment rule.

${ }^{18}$ We relax the assumption of complete information in Section 4.2
} 
tial supplier indicates a price at which he is willing to provide the whole supply contract. For simplicity we impose the feasibility condition that the suppliers' prices cannot exceed the budget, that is, providers choose their prices from the set $S=[0, b]$. Given the providers' prices, say $P=\left(p_{\ell}, p_{\mathfrak{f}}\right)$, the buyer determines the share of the supply contract assigned to each provider. To do so the buyer uses an allocation function $\varphi$ that associates to each vector of prices $P$ a vector $\varphi(P) \in \mathbb{R}_{+}^{2}$ such that $\varphi_{\ell}(P)+\varphi_{\mathfrak{f}}(P)=1$. We will introduce shortly two specific allocation functions.

Given an allocation function $\varphi$ and a vector of prices $P=\left(p_{\ell}, p_{\mathfrak{f}}\right)$, the profit of provider $i$ follows

$$
\pi_{i}(P)=\varphi_{i}(P)\left(p_{i}-c_{i}\right) .
$$

Equation (1) simply says that provider $i$ 's profit equals his market share times his mark-up.

This completes the description of the normal form game $\Gamma=\{I, S, \pi, \varphi\}$, where the set of agents is $I=\{\ell, \mathfrak{f}\}$, each agent's strategy space is $S \subseteq[0, b]$, each provider's profit is given by $\pi_{i}$, and the allocation function is $\varphi$. Our equilibrium concept is Nash equilibrium in pure strategies.

\subsection{The Buyer's Procurement Objectives}

In what follows we will introduce a specific allocation function, combine it with affirmative action, and evaluate the resulting equilibrium from the view point of the buyer. To model the buyer's objectives we follow Alcalde and Dahm (2013, 2019) and make the benchmark assumption that she is only interested in minimizing procurement costs. As explained in the Introduction, procurement auctions are usually organized as first-price auctions and we think of the buyer as comparing the procedure that we propose in this paper to a standard first-price auction for the whole supply contract. Since our main result identifies circumstances in which procurement expenditure is lower with our procedure than with a standard auction, this has the implication that the buyer prefers to use our procedure, even if she does not value affirmative action in itself 19

Given a vector of prices $P$, the FPA allocates the whole supply contract to the provider asking for the lowest price, that is, for each agent $i$,

$$
\varphi_{i}^{F P}(P)=\left\{\begin{array}{ll}
1 & \text { if } p_{i}<p_{j} \\
\frac{1}{2} & \text { if } p_{i}=p_{j} \\
0 & \text { if } p_{i}>p_{j}
\end{array} .\right.
$$

It is well known that the first-price sealed-bid auction under complete information does not possess a pure strategy Nash equilibrium. One way to restore existence of equilibrium is to

\footnotetext{
${ }^{19}$ Alternatively, we could postulate an objective function for the buyer that specifies how she tradesoff cost effectiveness and a measure of the success of affirmative action, like supplier diversity or the local provider's profits. This would yield results that depend on the specific formalization of the buyer's objectives. Since valuing for example supplier diversity would provide additional incentives for our procedure, the condition in our main result would be relaxed. Moreover, if the buyer's objective function is monotonic in supplier diversity, this condition might be relaxed quite substantially, because the optimal policy induces very substantial supplier diversity.
} 
make the realistic assumption that the providers' prices must be stated in legal tenders, so that the strategy space has a finite grid, that is, $S^{G}=\left\{p_{i} \in[0, b]: 100 p_{i} \in \mathbb{N}\right\}$. Alcalde and Dahm (2011) analyse pure strategy undominated Nash equilibria in the normal form game $\Gamma^{F P}=$ $\left\{I, S^{G}, \pi, \varphi^{F P}\right\}$ and show that in equilibrium the whole supply contract is ordered the price $c_{\ell}$ from the foreign provider ${ }^{20}$ Hence, our benchmark of comparison is the FPA that implies procurement costs of $C^{F P}=c_{\ell}{ }^{21}$

\subsection{The Contested Procurement Auction}

We now introduce the CPA (Alcalde and Dahm, 2013). Unlike the FPA, it has the virtue that the providers' shares are a smooth function of the prices. Given a vector of prices $P$, the share of provider $i$ is given by

$$
\varphi_{i}^{C P}(P)=\left\{\begin{array}{cl}
\frac{b+p_{j}-2 p_{i}}{2\left(b-p_{i}\right)} & \text { if } p_{i} \leq p_{j} \\
\frac{b-p_{i}}{2\left(b-p_{j}\right)} & \text { if } p_{i}>p_{j}
\end{array},\right.
$$

for $b>\min \left\{p_{i}, p_{j}\right\}$ and $\varphi_{i}^{C P}(P)=1 / 2$ for $p_{i}=p_{j}=b$.

Alcalde and Dahm (2013) analyse the normal form game $\Gamma^{C P}=\left\{I, S, \pi, \varphi^{C P}\right\}$ and show that in the unique equilibrium $\widehat{P}$ the providers' prices are

$$
\widehat{p}_{\ell}=\frac{b+c_{\ell}}{2} \quad \text { and } \quad \widehat{p}_{\mathfrak{f}}=b-\sqrt{\frac{\left(b-c_{\ell}\right)\left(b-c_{\mathfrak{f}}\right)}{4}} .
$$

Moreover, when the cost difference of the providers is large enough, that is,

$$
\frac{c_{\ell}-c_{f}}{b-c_{\ell}}>\left(\frac{13}{8}+\frac{5}{8} \sqrt{17}\right) \approx 4.20
$$

then the buyer's equilibrium provision cost under the CPA, denoted by $C^{C P}$, is lower than $C^{F P}=$ $c_{\ell}$, the cost under the FPA. The costs under the two allocation procedures are illustrated in Figure 1.

\footnotetext{
${ }^{20}$ For instance, Moldovanu and Sela (2003, footnote 12) write that "asymmetric Bertrand games (and first-price auctions) have no equilibria in pure strategies here, but introducing a smallest money unit immediately yields the intuitive solution." This intuitive solution in which the provider with the lowest cost wins the supply contract at a price equal to the other supplier's cost is the unique equilibrium if the grid size is fine enough. See Alcalde and Dahm (2011) for a general analysis and discussion of the FPA with finite grid.

${ }^{21}$ In the following subsections we introduce the CPA with affirmative action in form of a subsidy for the local provider. This raises the question of why we do not use the FPA with subsidy as a benchmark of comparison. Introducing a subsidy into the FPA benefits the buyer, as it allows to obtain provision costs arbitrary close (but higher) than $c_{\mathfrak{f}}$. Rather than implementing affirmative action, the buyer takes advantage of the presence of the local provider to appropriate (in the limit) all the surplus from the foreign supplier. The outcome is the same as a take it or leave it offer. Since this does not seem to be a good description of procurement auctions, we use the FPA without subsidy as our benchmark of comparison.
} 


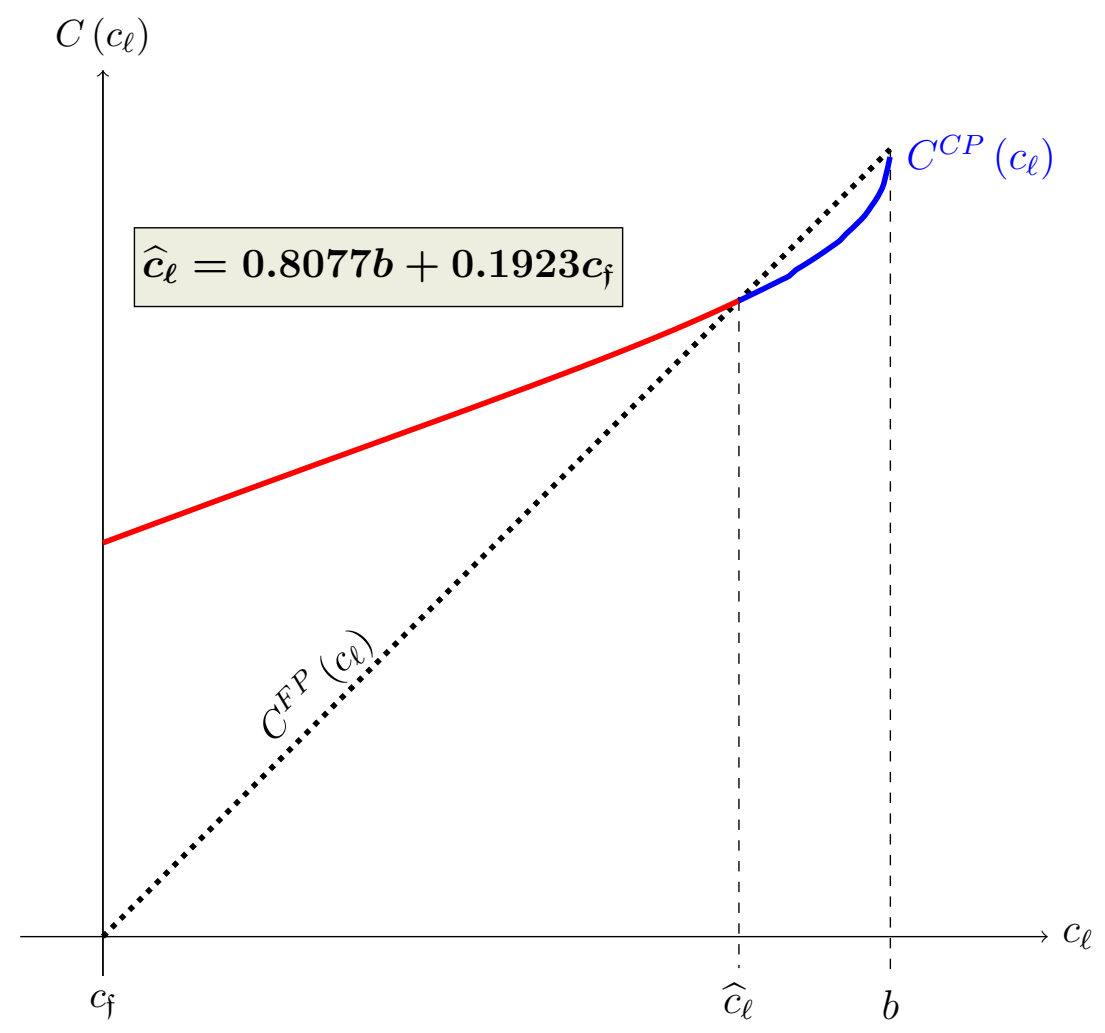

Figure 1: The Buyer's Provision Cost: FPA vs. CPA

\subsection{Affirmative Action}

In our benchmark model we consider affirmative action by means of subsidizing the local provider ${ }^{22}$ Instead of the local supplier's price $p_{\ell}$, the buyer pays a higher price $\alpha p_{\ell}$, with $\alpha>1$. Under such a scheme the local provider's profit function is increased by a premium, while the one of the foreign supplier remains as in (1), that is,

$$
\pi_{i}^{\alpha}\left(p_{\ell}, p_{\mathfrak{f}}\right)=\left\{\begin{array}{ll}
\varphi_{i}(P)\left(\alpha p_{i}-c_{i}\right) & \text { if } i=\ell \\
\varphi_{i}(P)\left(p_{i}-c_{i}\right) & \text { if } i=\mathfrak{f}
\end{array} .\right.
$$

The subsidy parameter $\alpha>1$ measures the intensity of affirmative action, as the higher $\alpha$, the more intense the program ${ }^{23}$ The buyer announces the intensity of affirmative action before the providers indicate their prices.

Since the subsidy increases the price of the local provider by a premium, the price including the affirmative action premium might exceed the budget and hence not be feasible. To avoid

\footnotetext{
${ }^{22}$ In Subsection 4.1 we show that the same results are obtained under the alternative assumption that the allocation function $\varphi$ is biased in favour of the local supplier.

${ }^{23}$ The intensity of the program can be measured with the function $I^{S}(\alpha)=\alpha-1$. For ease of the exposition, however, we will refer in later sections to $\alpha$ (rather than to $\alpha-1$ ) as the intensity of the program.
} 
this we assume the following safeguard clause: the local provider obtains the (entire) affirmative action premium only if $\alpha p_{\ell} \leq b \sqrt{24}$ We model this by describing the profits of the local provider as

$$
\pi_{\ell}^{\alpha C}\left(p_{\ell}, p_{\mathfrak{f}}\right)=\varphi_{\ell}\left(p_{\ell}, p_{\mathfrak{f}}\right)\left(p_{\ell}^{e}-c_{\ell}\right),
$$

where the 'effective' price for the local provider is $p_{\ell}^{e}=\min \left\{\alpha p_{\ell}, b\right\}$. This defines the normal form game $\Gamma^{\alpha C}=\left\{I, S, \pi^{\alpha C}, \varphi\right\}$. It differs from $\Gamma$ only in that the local provider's profit function is given by $\pi_{\ell}^{\alpha C}$ defined in $(7)$. The foreign provider's profit function $\pi_{\mathfrak{f}}$ remains unchanged and follows (1). In our benchmark model in Section 3 we focus on the CPA defined in (3) and analyse $\Gamma^{C P \alpha C}=\left\{I, S, \pi^{\alpha C}, \varphi^{C P}\right\}$. In what follows we refer to $\Gamma^{C P \alpha C}$ as the CPA with subsidy $\alpha$.

\section{Contested Procurement with Affirmative Action}

In this section we focus on the CPA with subsidy $\alpha$. Our analysis is organised as follows. First, Subsection 3.1 establishes existence and uniqueness of equilibrium in the CPA with subsidy $\alpha$. We turn then in Subsection 3.2 to the distributive consequences of affirmative action and show that increasing the intensity $\alpha$ of the program improves the position of the local provider. Lastly, Subsection 3.3 provides a condition under which the appropriate intensity of affirmative action in the CPA not only guarantees very substantial supplier diversity, but also reduces the buyer's provision cost compared to the equilibrium of a first-price auction.

\subsection{Equilibrium}

The first result in this section characterizes the unique equilibrium in closed-form for any intensity $\alpha$ of the subsidy. Before stating this result we introduce the notation $H\left(b, c_{\mathfrak{f}}\right) \equiv 2 b c_{\mathfrak{f}} /\left(b+c_{\mathfrak{f}}\right)$ for the harmonic mean of $c_{\mathfrak{f}}$ and $b 25$

Theorem 1 The CPA with subsidy $\alpha>1$ has a unique equilibrium $\left(p_{\ell}^{*}, p_{\mathfrak{f}}^{*}\right)$ described as follows.

\footnotetext{
${ }^{24}$ To be fully precise, since the local supplier only produces a share of the supply contract, $\alpha p_{\ell} \leq b$ is a sufficient but not a necessary condition to avoid that the price including the affirmative action premium exceeds the budget. We use this formalization of the safeguard clause for simplicity. We will show that this formalization of the safeguard clause allows us to derive an equivalence result between the equilibrium of an affirmative action program affecting the award rule and the equilibrium of a program providing a subsidy, see Subsection 4.1 .

${ }^{25}$ Although we are primarily interested in situations with affirmative action in which $\alpha>1$ holds, we remark that the statement also holds for the symmetric setting in Alcalde and Dahm (2013) where $\alpha=1$. For $\alpha=1$ the first row in both part (a) and part (b) are relevant and prescribe the same equilibrium prices. The statement of the theorem distinguishes six intervals of the affirmative action intensity $\alpha$. At the end of the proof of Theorem 1 we describe these intervals explicitly.
} 
(a) If the cost difference of providers is large, that is, $c_{\ell} \geq H\left(b, c_{\mathfrak{f}}\right)$, then

$$
\left(p_{\ell}^{*}, p_{\mathfrak{f}}^{*}\right)=\left\{\begin{array}{cl}
\left(\frac{\alpha b+c_{\ell}}{2 \alpha}, b-\sqrt{\left.\frac{\left(b-c_{\mathfrak{f}}\right)\left(\alpha b-c_{\ell}\right)}{4 \alpha}\right)}\right) & \text { if } \alpha \leq \frac{2 b-c_{\ell}}{b} \\
\left(\frac{b}{\alpha}, b-\sqrt{\frac{(\alpha-1) b\left(b-c_{\mathfrak{f}}\right)}{2 \alpha}}\right) & \text { if } \frac{2 b-c_{\ell}}{b}<\alpha \leq \frac{2 b}{b+c_{\mathfrak{f}}} \\
\left(\frac{b}{\alpha}, \frac{b+c_{\mathfrak{f}}}{2}\right) & \text { if } \alpha>\frac{2 b}{b+c_{\mathfrak{f}}}
\end{array}\right.
$$

(b) If the cost difference of providers is small, that is, $c_{\ell}<H\left(b, c_{\mathfrak{f}}\right)$, then

$$
\left(p_{\ell}^{*}, p_{\mathfrak{f}}^{*}\right)=\left\{\begin{array}{cl}
\left(\frac{\alpha b+c_{\ell}}{2 \alpha}, b-\sqrt{\frac{\left(b-c_{\mathfrak{f}}\right)\left(\alpha b-c_{\ell}\right)}{4 \alpha}}\right) & \text { if } \alpha c_{\mathfrak{f}} \leq c_{\ell} \\
\left(\min \left\{b-\sqrt{\frac{\left(b-c_{\mathfrak{f}}\right)\left(\alpha b-c_{\ell}\right)}{4 \alpha}}, \frac{b}{\alpha}\right\}, \frac{b+c_{\mathfrak{f}}}{2}\right) & \text { if } \alpha c_{\mathfrak{f}}>c_{\ell}
\end{array}\right.
$$

Since the harmonic mean partitions the interval $\left[c_{f}, b\right]$ into two sub-intervals, the theorem distinguishes between situations in which the cost difference of providers is large (part (a)) and those in which it is small (part(b)). In both cases affirmative action levels the playing field by making the local provider more competitive and the foreign supplier reacts optimally to this. Affirmative action with low intensity does not affect equilibrium behaviour much. The local provider behaves as if his cost were reduced to $c_{\ell} / \alpha$ instead of $c_{\ell}$ and chooses the midpoint between this value and $b$. As in the situation without subsidy (Alcalde and Dahm, 2013), his optimal price does not depend on the foreign provider's cost and the foreign provider undercuts his rival's price optimally, trading-off market share and mark-up.

When the cost difference of providers is large (part (a) and affirmative action is sufficiently intense, the safeguard clause kicks in and the local provider caps his price at $b / \alpha$. But since raising the intensity of affirmative action further decreases $b / \alpha$, the local provider is eventually strengthened enough to undercut his rival. At that point the foreign provider behaves as the high-cost provider and chooses the midpoint between his cost and $b$, so that his price is independent of his rival's price.

The case in which the cost difference of providers is small (part (b)) differs from the situation with large cost difference (part (a) in that affirmative action can induce providers to switch the roles of high-cost and low-cost providers before the safeguard clause kicks in. When the intensity of affirmative action increases sufficiently, the foreign supplier behaves as a high-cost provider and his optimal price does not depend on the local provider's cost. The local provider in turn acts as a low-cost supplier and undercuts his rival's price optimally. Raising the intensity of affirmative action further, the safeguard clause becomes binding and the local provider caps his price at $b / \alpha$, while the foreign supplier's price is unchanged.

Note that the equilibrium prices in Theorem 1 are continuous functions of the affirmative action intensity $\alpha$. We make this explicit using the notation $\left(p_{\ell}^{*}, p_{\mathfrak{f}}^{*}\right)=\left(p_{\ell}(\alpha), p_{\mathfrak{f}}(\alpha)\right)$. Based 
on this notation we define for later reference the following thresholds for $\alpha$ that have already proved important in the statement of Theorem 1. First, we define the unique intensity level $\alpha^{*}$ for which at equilibrium the safeguard clause becomes binding as

$$
\alpha^{*}=\sup \left\{\alpha \geq 1: \alpha p_{\ell}(\alpha)<b\right\} \text {. }
$$

Theorem 1 implies that the mathematical expression for $\alpha^{*}$ differs depending on whether the cost difference of providers is large or small. ${ }^{26}$ Second, we define the unique intensity $\alpha^{e}$ for which the equilibrium prices of both providers are equal, that is, $p_{\ell}\left(\alpha^{e}\right)=p_{\mathfrak{f}}\left(\alpha^{e}\right)$. This 'equalizing' or 'level playing field' intensity is given by

$$
\alpha^{e}=\left\{\begin{array}{cl}
\frac{2 b}{b+c_{\mathfrak{f}}} & \text { if } c_{\ell} \geq H\left(b, c_{\mathfrak{f}}\right) \\
\frac{c_{\ell}}{c_{\mathfrak{f}}} & \text { if } c_{\ell}<H\left(b, c_{\mathfrak{f}}\right)
\end{array} .\right.
$$

Notice that $\alpha^{e}$ is a continuous function of $c_{\ell}$.

We conclude this subsection illustrating the definitions of $\alpha^{*}$ and $\alpha^{e}$ as well as the equilibrium prices in Theorem 1 with the following example.

Example 1 Consider the following three procurement problems $\left(b_{1}, c_{\ell}, c_{\mathfrak{f}}\right)=(95,90,72)$, $\left(b_{2}, c_{\ell}, c_{\mathfrak{f}}\right)=(110,90,72)$ and $\left(b_{3}, c_{\ell}, c_{\mathfrak{f}}\right)=(130,90,72)$. Notice that these problems differ only in the size of the budget. Since the harmonic mean is increasing in its arguments, part (a) of Theorem 1 applies to the first two problems and part (b) to the third problem, as $H_{1}\left(b_{1}, c_{\mathfrak{f}}\right) \simeq 81.92$, $H_{2}\left(b_{2}, c_{\mathfrak{f}}\right) \simeq 87.03$ and $H_{3}\left(b_{3}, c_{\mathfrak{f}}\right) \simeq 92.67$. The thresholds at which the safeguard clause becomes binding are $\alpha_{1}^{*} \simeq 1.05, \alpha_{2}^{*} \simeq 1.18$ and $\alpha_{3}^{*} \simeq 1.3$, while the 'equalizing' intensity levels are given by $\alpha_{1}^{e} \simeq 1.14, \alpha_{2}^{e} \simeq 1.21$ and $\alpha_{3}^{e}=1.25$. Figure 2 shows the equilibrium prices for the three problems as functions of $\alpha$. Each problem corresponds to a pair of functions that intersect; the higher the budget the higher the position of the pair of functions. The vertical grid lines indicate $\alpha^{*}$ and $\alpha^{e}$ for each problem. In all three problems the equilibrium prices are piecewise-defined but continuous functions of the intensity $\alpha$ and a sufficiently high level of intensity induces the local provider to undercut his rival's price.

To understand why the budget constraint affects the providers' equilibrium prices, recall the CPA allocation procedure when the local provider sets a higher price than the foreign supplier (the second line in equation (3)). When deciding whether to reduce his price the local supplier trades-off the marginal cost of this adjustment in form of a smaller mark-up with the marginal benefit in form of a larger share. Affirmative action subsidises the marginal costs, inducing him to set a more competitive price. Consequently, the functions $p_{\ell}$ in Figure 2 are decreasing in $\alpha$. A larger budget, however, reduces the marginal benefits. As a result, the equilibrium prices in Figure 2 shift upwards as the budget increases.

\footnotetext{
${ }^{26}$ In the former case we have that $\alpha^{*}=\left(2 b-c_{\ell}\right) / b$. For completeness we include the algebraic expression for the latter case in Appendix A.1 in equation (31). For ease of the exposition, we omit the arguments of the function $\alpha^{*}\left(b, c_{\ell}, c_{\mathfrak{f}}\right)$ when they are clear from the context and write simply $\alpha^{*}$. We follow the same convention with $\alpha^{e}$ which we define next.
} 


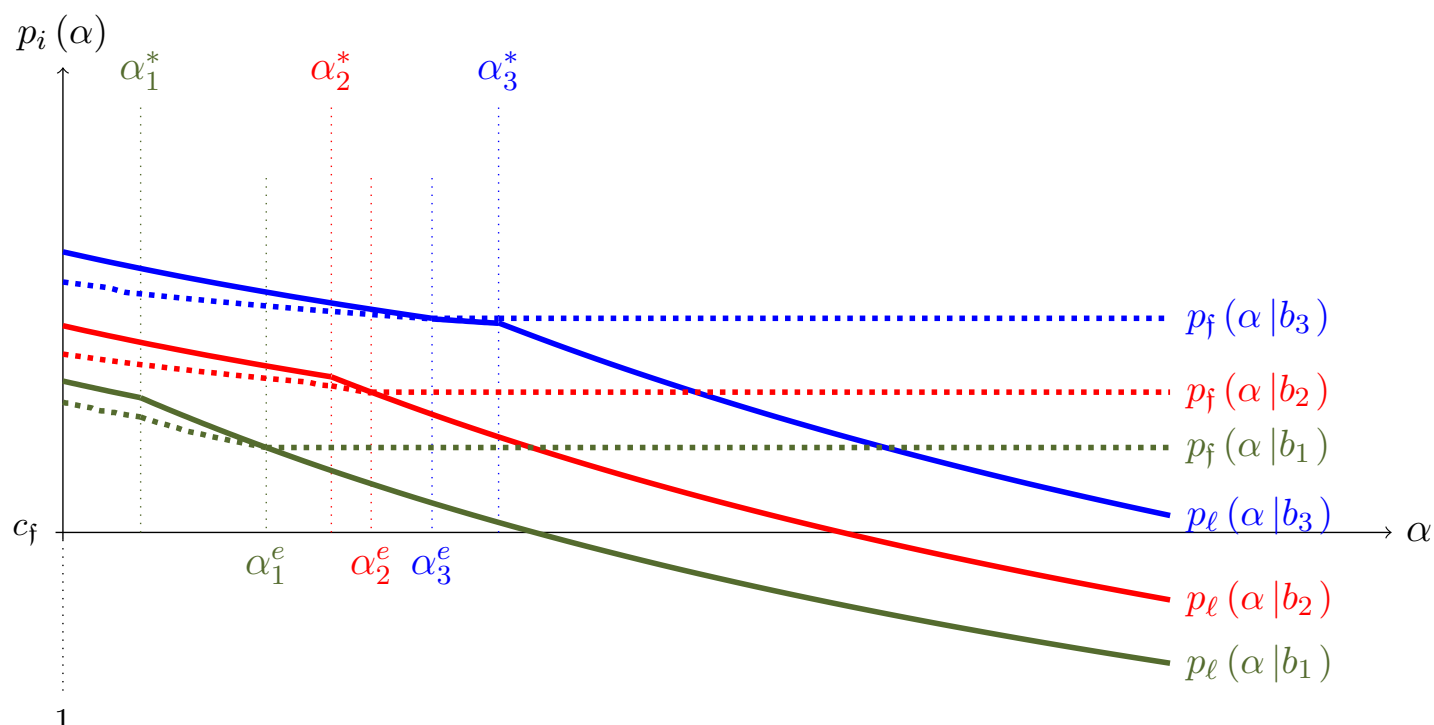

Figure 2: Equilibrium Prices

\subsection{The Distributive Consequences of Affirmative Action}

We now turn to the distributive consequences of affirmative action. Since the objective of such a policy is to favour the local provider, it is important to understand to what extent affirmative action policies reach this aim.

There are several related ways to measure the distributive consequences. First, one might look at the provision share of the local provider, that is, supplier diversity. As explained in the Introduction, affirmative action policies in the context of U.S. government contracts might specify target market shares for women-owned businesses, minority-owned businesses, small businesses, disabled-owned businesses, veteran-owned businesses and others. For a given intensity $\alpha$, taking into account the equilibrium prices $\left(p_{\ell}^{*}, p_{\mathfrak{f}}^{*}\right)=\left(p_{\ell}(\alpha), p_{\mathfrak{f}}(\alpha)\right)$ described in Theorem $[1$, the equilibrium provision shares are given by

$$
\sigma_{\ell}(\alpha)=\varphi_{\ell}^{C P}\left(p_{\ell}(\alpha), p_{\mathfrak{f}}(\alpha)\right) \quad \text { and } \quad \sigma_{\mathfrak{f}}(\alpha)=1-\sigma_{\ell}(\alpha) .
$$

Second, since the local provider's revenue is given by the buyer's share of expenditure on this provider, one might also investigate how this share of expenditure varies with the affirmative action intensity. Let $C_{i}(\alpha)$ denote the buyer's equilibrium payment to provider $i$ when a program with intensity $\alpha$ is implemented. Total expenditure $C(\alpha)$ is given by $C(\alpha)=C_{\ell}(\alpha)+C_{\mathfrak{f}}(\alpha)$, where

$$
C_{\ell}(\alpha)=\alpha p_{\ell}(\alpha) \sigma_{\ell}(\alpha) \text { and } \quad C_{\mathfrak{f}}(\alpha)=p_{\mathfrak{f}}(\alpha) \sigma_{\mathfrak{f}}(\alpha) .
$$

Third, one might analyse how equilibrium profits vary with the affirmative action intensity. Let $\Pi_{i}(\alpha)$ denote equilibrium profits. Since the local provider has an incentive to avoid that the safeguard clause kicks in, equilibrium profits can be described as

$$
\Pi_{\ell}(\alpha)=\sigma_{\ell}(\alpha)\left(\alpha p_{\ell}(\alpha)-c_{\ell}\right) \quad \text { and } \quad \Pi_{\mathfrak{f}}(\alpha)=\sigma_{\mathfrak{f}}(\alpha)\left(p_{\mathfrak{f}}(\alpha)-c_{\mathfrak{f}}\right) .
$$


The following result establishes how these three measures vary with the intensity of affirmative action.

Proposition 1 In the CPA with subsidy $\alpha>1$, as the intensity $\alpha$ of the affirmative action program increases,

(a) the equilibrium provision share $\sigma_{\ell}(\alpha)$, the revenue $C_{\ell}(\alpha)$ and the profits $\Pi_{\ell}(\alpha)$ of the local provider increase, while

(b) the equilibrium provision share $\sigma_{\mathfrak{f}}(\alpha)$, the revenue $C_{\mathfrak{f}}(\alpha)$ and the profits $\Pi_{\mathfrak{f}}(\alpha)$ of the foreign supplier decrease.

We illustrate Proposition 1 with the procurement problems from Example 1. Since the three measures of distributive consequences have similar comparative statics and since the next subsection deals with the buyer's provision costs which are the sum of the providers' revenues, we focus on revenues.

Example 2 Consider again the three procurement problems $\left(b_{1}, c_{\ell}, c_{\mathfrak{f}}\right)=(95,90,72)$, $\left(b_{2}, c_{\ell}, c_{\mathfrak{f}}\right)=(110,90,72)$ and $\left(b_{3}, c_{\ell}, c_{\mathfrak{f}}\right)=(130,90,72)$ from Example 1. Figure 3 shows the providers' revenues for the three problems as functions of $\alpha$. In all three problems the suppliers' revenues are piecewise-defined but continuous and monotonic functions of the intensity $\alpha$.

Notice that the CPA is very sensitive to the intensity $\alpha$ of the affirmative action program. Any increase in intensity improves the position of the local provider no matter how the distributive consequences are measured. This is not the case with the FPA, where the local provider's share is zero unless the intensity level of the affirmative action program is sufficient to level the playing field completely. The fact that increasing the intensity of the affirmative action program results in a transfer of revenue from the foreign provider to the local supplier does not imply that total provision costs are constant. In fact, in the next subsection we show that raising the intensity of affirmative action might even decrease provision costs below $c_{\ell}$. This contrasts again with the FPA where it is not possible to successfully induce a substantial share for the high-cost supplier (that is, the local firm wins) and have provision costs below $c_{\ell}$ (as $\alpha p_{\ell}<c_{\ell}$ implies that the local firm makes losses).

\subsection{Procurement Expenditure with Affirmative Action}

In this section we explore how provision costs depend on the intensity of affirmative action. As explained in the Introduction, this is an important question, because there is a popular perception that affirmative action programs increase provision costs. Note that, even though Proposition 1 does not address this issue directly, it does suggest the possibility that this popular perception might be incorrect. This would be the case if for some intensities of the program, the foreign supplier's revenue decreases faster than the local provider's revenue increases. The following example illustrates this idea. 


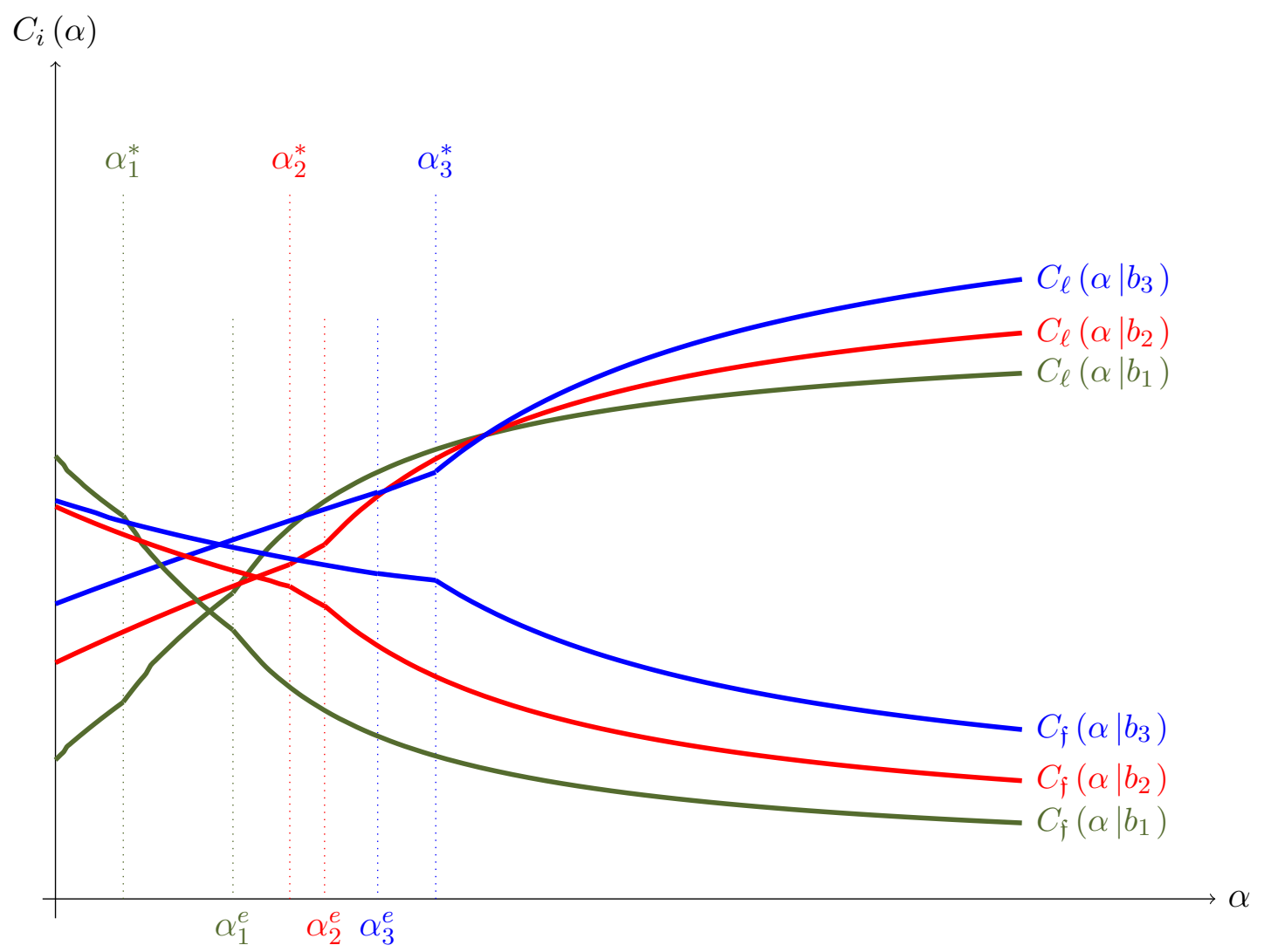

Figure 3: Expenditure Shares of Suppliers

Example 3 Consider again the three procurement problems $\left(b_{1}, c_{\ell}, c_{\mathfrak{f}}\right)=(95,90,72)$, $\left(b_{2}, c_{\ell}, c_{\mathfrak{f}}\right)=(110,90,72)$ and $\left(b_{3}, c_{\ell}, c_{\mathfrak{f}}\right)=(130,90,72)$ from Examples 1 and 2 . Figure 4 shows the buyers' provision costs for the three problems as functions of $\alpha{ }^{27}$ In the first two problems there is a local minimum at $\alpha^{e}$, while in the third problem provision costs are increasing in $\alpha$. In the first problem this local minimum is also global, implying that for a wide variety of intensities affirmative action does not increase provision costs. In fact, at this global minimum provision costs are even below $c_{\ell}$, the provision costs arising from a standard FPA without affirmative action.

The purpose of the remainder of this subsection is to generalise the previous example. We investigate under what conditions affirmative action reduces provision costs and describe which levels of intensity are optimal. We first establish in Proposition 2 a limit on the intensity of the affirmative action program. It is never beneficial to exceed $\alpha^{e}$, the level for which the equilibrium prices and shares of both providers are equal. This implies that $\sigma_{\ell}$ the share allocated to the local provider, should never exceed half of the supply contract. We then explore in Theorem 2 conditions under which an affirmative action program (with small but positive intensity) reduces

\footnotetext{
${ }^{27}$ Unlike Figure 1 , in this section we focus on the CPA. Hence for simplicity we omit the superindex $C P$ and denote the cost function by $C(\cdot)$.
} 


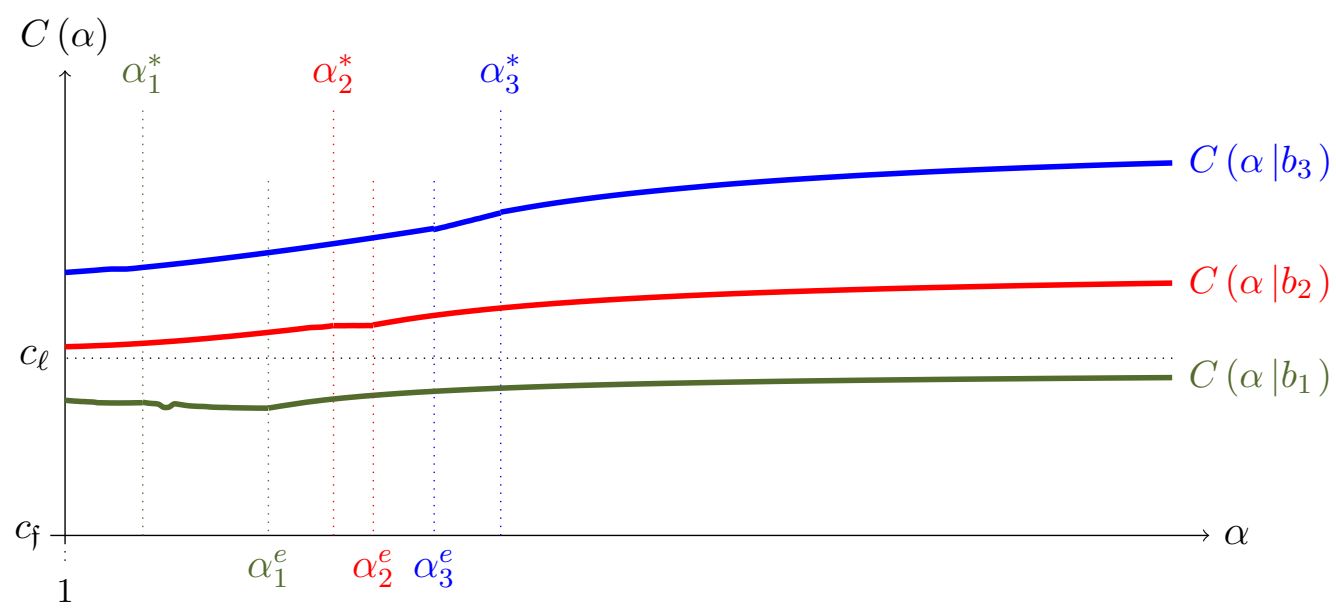

Figure 4: Provision Costs

the buyers' provision cost, compared to the benchmark without affirmative action. Lastly, we provide in Theorem 3 conditions that guarantee that the intensity $\alpha^{e}$ for which the equilibrium prices and shares of both providers are equal is both a local and global minimiser of provision costs. In the later case, when the providers' costs are different enough, provision costs are lower than $c_{\ell}$, the provision costs arising from a standard FPA without affirmative action. The conditions in Theorems 2 and 3 essentially require the cost difference of providers to be large enough.

We start establishing that it is never beneficial to choose an intensity of affirmative action exceeding $\alpha^{e}$, the level for which the equilibrium prices and shares of both providers are equal as defined in (9).

Proposition 2 In the CPA with subsidy $\alpha>1$, the total provision cost function $C(\alpha)$ is increasing in the affirmative action intensity $\alpha$ whenever it exceeds $\alpha^{e}$.

The intuition for this result is as follows. When the intensity is $\alpha^{e}$ the equilibrium prices and shares of both providers are equal, that is, $\sigma_{\ell}\left(\alpha^{e}\right)=\sigma_{\mathfrak{f}}\left(\alpha^{e}\right)=0.5$. When the intensity of the program increases further so that it exceeds $\alpha^{e}$, the equilibrium price of the foreign supplier remains the same. The local provider, however, reduces his price $p_{\ell}(\alpha)$, increasing thereby his share of the supply contract. At the same time his 'effective price' $\alpha p_{\ell}(\alpha)$ is non-decreasing in the intensity. Therefore, total provision costs are increasing in the intensity when the intensity exceeds $\alpha^{e}$.

We now turn to low intensity affirmative action programs and provide a condition under which the introduction of such a program reduces provision costs, compared to the benchmark without affirmative action. To do so consider a procurement problem $\left(b, c_{\ell}, c_{\mathfrak{f}}\right)$ and define the function $\beta\left(b, c_{\ell}, c_{\mathfrak{f}}\right)$ as follows

$$
\beta\left(b, c_{\ell}, c_{\mathfrak{f}}\right)=\frac{2 b-c_{\ell}}{2} \sqrt{\frac{b-c_{\ell}}{b-c_{\mathfrak{f}}}}-c_{\ell}\left(\sqrt{\frac{b-c_{\mathfrak{f}}}{b-c_{\ell}}}-1\right) .
$$


Theorem 2 There is an intensity $\alpha>1$ for the CPA with subsidy such that $C(\alpha)<C(1)$ if and only if $\beta\left(b, c_{\ell}, c_{\mathfrak{f}}\right)<0$.

To gain an intuition for this result notice that the function $\beta\left(b, c_{\ell}, c_{\mathfrak{f}}\right)$ measures the marginal provision costs when a low intensity affirmative action program is introduced, that is, $\alpha \rightarrow 1^{+}$. Intuitively, if the slope of $C(\alpha \mid b)$ in Figure 4 at that point is negative then there exists some intensity of affirmative action that is beneficial for the buyer.

It turns out that the condition $\beta\left(b, c_{\ell}, c_{\mathfrak{f}}\right)<0$ is the easier to be fulfilled the smaller $b$, the larger $c_{\ell}$, and the smaller $c_{\mathrm{f}}{ }^{28}$ This implies this condition will hold when the cost difference of providers is sufficiently large. To see the relationship between the cost difference and the budget, remember that Theorem 1 measured the cost difference comparing the harmonic mean $H\left(b, c_{\mathfrak{f}}\right)$ to $c_{\ell}$. A competitive budget implies that the cost difference is large in this sense, as for $b \rightarrow c_{\ell}^{+}$, we have that $c_{\ell}>H\left(b, c_{\mathfrak{f}}\right) .29$

We turn now to our main result, which considers the properties of the provision cost function at $\alpha^{e}$. This is the 'equalizing' or 'level playing field' intensity for which the equilibrium prices and shares of both providers are equal. We have the following result.

Theorem 3 Let the cost difference of providers be large, that is, $c_{\ell} \geq H\left(b, c_{\mathfrak{f}}\right)$. Then, in the CPA with subsidy $\alpha>1$, the buyer's total provision costs $C(\alpha)$ have a (local) minimum at $\alpha^{e}=2 b /\left(b+c_{\mathfrak{f}}\right)$. If, in addition,

$$
\frac{c_{\ell}-c_{\mathfrak{f}}}{b-c_{\ell}}>3
$$

holds, then $\alpha^{e}$ is a global minimizer and $C\left(\alpha^{e}\right)<c_{\ell}$.

Several comments are in order. First, Theorem 3 says that choosing the intensity of affirmative action in the CPA optimally, the buyer can reconcile supplier diversity and cost effectiveness. Supplier diversity is maximal in the sense that affirmative action levels the playing field completely. As a result equilibrium prices and shares of both providers are equal. In addition, when condition (11) holds, the supply contract is allocated in a cost effective way. This is because provision costs are lower than $c_{\ell}$, the provision costs arising from a standard FPA without affirmative action.

Second, notice that condition (11) is less restrictive than condition (5), which was derived in our earlier paper Alcalde and Dahm (2013) for the CPA without affirmative action. Similar

\footnotetext{
${ }^{28}$ To be fully precise, the function $\beta\left(b, c_{\ell}, c_{\mathfrak{f}}\right)$ is increasing in $b$ and $c_{\mathfrak{f}}$, while the derivative with respect to $c_{\ell}$ is in general ambiguous. However, inspection of (10) shows that $\beta>0$ for $c_{\ell} \rightarrow c_{\mathrm{f}}$ and $\beta \rightarrow-\infty$ for $c_{\ell} \rightarrow b$. In addition, the function $\beta\left(b, c_{\ell}, c_{\mathfrak{f}}\right) / c_{\ell}$ is decreasing in $c_{\ell}$. This implies that the condition $\beta\left(b, c_{\ell}, c_{\mathfrak{f}}\right)<0$ is fulfilled when $c_{\ell}$ is sufficiently large.

${ }^{29}$ The fact that $c_{\ell} \geq H\left(b, c_{\mathfrak{f}}\right)$ does not imply that $\beta\left(b, c_{\ell}, c_{\mathfrak{f}}\right)<0$. To see this remember the three procurement problems $\left(b_{1}, c_{\ell}, c_{\mathfrak{f}}\right)=(95,90,72),\left(b_{2}, c_{\ell}, c_{\mathfrak{f}}\right)=(110,90,72)$ and $\left(b_{3}, c_{\ell}, c_{\mathfrak{f}}\right)=(130,90,72)$ from Examples 1 3. On the one hand, we know already from Example 1 that in the first two problems $c_{\ell} \geq H\left(b, c_{\mathfrak{f}}\right)$ holds. On the other hand, the value of $\beta_{t}=\beta\left(b_{t}, 90,72\right)$ increases in the budget constraint from $\beta_{1} \simeq-79.72$, to $\beta_{2} \simeq 13.1$ to $\beta_{3} \simeq 52.22$ and equals zero for $b \simeq 105.82$. Hence, for the second problem, we have $c_{\ell}>H\left(b_{2}, c_{\mathfrak{f}}\right)$ and $\beta_{2}>0$.
} 
to the condition in Theorem 2, it holds when the cost difference of providers is sufficiently large, as then in the expression on the left hand side of (11) the numerator is large, while the denominator is small. Of course, when

$$
4.2>\frac{c_{\ell}-c_{\mathfrak{f}}}{b-c_{\ell}}>3
$$

holds, then complementing the CPA with affirmative action is crucial for the cost effectiveness of the CPA. This is so, because in the setting of Alcalde and Dahm (2013) provision costs are higher than $c_{\ell}$, while the opposite is true when affirmative action levels the playing field completely.

Finally, notice that the intensity level of affirmative action needed in the CPA to level the playing field completely is lower than the level needed in the FPA to induce the local firm to win the contract 30

\section{Extensions}

The existence of an equilibrium with the potential to reconcile the conflicting aims of cost effective procurement and supplier diversity continues to hold under varying conditions. This section considers several extensions. We consider an affirmative action program affecting the award rule and private information. We also discuss how our setting might be extended to multiple sourcing with affirmative action.

\subsection{Other Affirmative Action Policies}

In this subsection we provide an equivalence result between the equilibrium of an affirmative action program affecting the award rule and the equilibrium of a program providing a subsidy. The existence of this equivalence is intuitive and well known in related bidding games, including contests (Szidarovszky and Okuguchi, 1997; Esteban and Ray, 1999) and auctions (Athey et al., 2013) ${ }^{31}$ Surprisingly, in our setting there are intricacies of this equivalence that come from the fact that -unlike in standard bidding games- the providers' strategy spaces are bounded.

\subsubsection{The Setting}

We generalize the setting in Section 3 in several respects. First, we allow for strategy spaces with a finite grid, that is, $p_{i} \in S \subseteq[0, b]$. Second, we consider allocation functions $\varphi$ fulfilling the following two incentive-compatibility properties:

(C.1) The share allocated to each provider $i$ is non-increasing on the supplier's price, that is, for each $P=\left(p_{i}, p_{j}\right), \varphi_{i}\left(p_{i}, p_{j}\right) \geq \varphi_{i}\left(p_{i}^{\prime}, p_{j}\right)$ whenever $p_{i}^{\prime}>p_{i}$.

\footnotetext{
${ }^{30}$ In the FPA this requires that the local provider can outbid the foreign supplier. Hence $c_{\mathfrak{f}}>c_{\ell} / \alpha$ or $\alpha>c_{\ell} / c_{\mathfrak{f}}$ must hold. Notice that $\alpha^{e}=2 b /\left(b+c_{\mathfrak{f}}\right)<c_{\ell} / c_{\mathfrak{f}}$ if and only if $c_{\ell}>H\left(b, c_{\mathfrak{f}}\right)$.

${ }^{31}$ In Appendix A.5 we clarify the relationship between our setting and contest games. We also compare our equivalence result in Lemma 1 below to its analogue for contest games.
} 
(C.2) The share function $\varphi$ is cross-monotonic, that is, for $P=\left(p_{i}, p_{j}\right)$ given, if $p_{i}<p_{j}$, then $\varphi_{i}(P)>\varphi_{j}(P)$.

Condition (C.1) says that a provider's share is monotonic in his price. The cross-monotonicity condition (C.2) relates the prices and shares of the two providers. It implies an equal treatment of equals or symmetry property saying that if the two providers choose the same price, their shares are equal 32

Our equivalence result relates the equilibrium of a subsidy program and the equilibrium of an affirmative action program affecting the award rule. The latter program transforms the (original) symmetric allocation function $\varphi$ into a biased allocation function $\varphi^{\delta}$ in the following way. Given a bias $\delta \in(0,1)$, for each vector of prices $\left(p_{\ell}, p_{\mathfrak{f}}\right)$, provider $i$ 's share is

$$
\varphi_{i}^{\delta}\left(p_{\ell}, p_{\mathfrak{f}}\right)=\varphi_{i}\left(\delta p_{\ell}, p_{\mathfrak{f}}\right),
$$

and thus his profit becomes

$$
\pi_{i}^{\delta}\left(p_{\ell}, p_{\mathfrak{f}}\right)=\varphi_{i}^{\delta}\left(p_{\ell}, p_{\mathfrak{f}}\right)\left(p_{i}-c_{i}\right)=\varphi_{i}\left(\delta p_{\ell}, p_{\mathfrak{f}}\right)\left(p_{i}-c_{i}\right) .
$$

This defines the normal form game $\Gamma^{\delta}=\left\{I, S, \pi^{\delta}, \varphi^{\delta}\right\}$. It differs from $\Gamma$ in that each provider's profit is given by $\pi^{\delta}$, and the allocation function is $\varphi^{\delta}$. In what follows we refer to $\Gamma^{\delta}$ as an affirmative action program with bias $\delta$.

For simplicity of the exposition in this section we focus on undominated Nash equilibria in pure strategies ${ }^{33}$ A strategy or price of provider $i$ in $\Gamma^{\delta}$ and of provider $\mathfrak{f}$ in $\Gamma^{\alpha C}$ is undominated if and only if $c_{i}<p_{i} \leq b$, while a strategy or price of provider $\ell$ in $\Gamma^{\alpha C}$ is undominated if and only if $c_{\ell} / \alpha<p_{\ell} \leq b / \alpha 34$

\subsubsection{The Equivalence Result}

Our next result establishes an equivalence between a program with bias $\delta$ and a program with subsidy $\alpha$ provided $\delta$ and $\alpha$ satisfy the natural condition $\alpha=1 / \delta$.

Before we introduce our result we need to take into account a (technical) complication arising from the fact that we allow for strategy spaces with a finite grid. We introduce a condition on the grid that allows to compare the strategies selected by the local provider in the two games.

\footnotetext{
${ }^{32} \mathrm{~A}$ rule that does not fulfil this condition, like e.g. the (constant) equal share rule defined as $\varphi\left(p_{\ell}, p_{\mathfrak{f}}\right)=(0.5,0.5)$ for any vector of prices, might lead to multiple equilibria, and the equivalence in Lemma 1 below becomes more complex.

${ }^{33}$ For completeness we state that $p_{\ell} \in S$ is a dominated price for $\ell$ whenever there is another price, say $p_{\ell}^{\prime}$ such that, for each $p_{\mathfrak{f}} \in S, \pi_{\ell}\left(p_{\ell}^{\prime}, p_{\mathfrak{f}}\right) \geq \pi_{\ell}\left(p_{\ell}, p_{\mathfrak{f}}\right)$, with the above inequality being strict for some price selected by provider $\mathfrak{f}$. Dominated prices for provider $\mathfrak{f}$ are described in a similar way.

${ }^{34}$ Notice that, for the local provider $\ell$, the price $b / \alpha$ dominates any price in $(b / \alpha, b]$. The reason is that for any given price of the foreign seller, say $p_{\mathfrak{f}}$, and any price of the local provider $p_{\ell}>b / \alpha$ the local provider's mark-up is the same no matter if he selects $p_{\ell}$ or $b / \alpha$, that is $\min \left\{\alpha p_{\ell}, b\right\}=b=$ $\min \{\alpha(b / \alpha), b\}$. By condition (C.1), $\varphi_{\ell}\left(p_{\ell}, p_{\mathfrak{f}}\right) \leq \varphi_{\ell}\left(b / \alpha, p_{\mathfrak{f}}\right)$, and thus $\pi_{\ell}^{\alpha C}\left(p_{\ell}, p_{\mathfrak{f}}\right) \leq \pi_{\ell}^{\alpha C}\left(b / \alpha, p_{\mathfrak{f}}\right)$. Now, for $p_{\ell}>b / \alpha$ consider $p_{\mathfrak{f}}=p_{\ell}$. Then, by condition (C.2), the above inequality on $\ell$ 's shares becomes strict, and thus $\pi_{\ell}^{\alpha C}\left(p_{\ell}, p_{\mathfrak{f}}\right)<\pi_{\ell}^{\alpha C}\left(b / \alpha, p_{\mathfrak{f}}\right)$ whenever $p_{\mathfrak{f}}=p_{\ell}>b / \alpha$.
} 
Assume that the 'common' strategy space is $S_{\ell} \subseteq[0, b]$, and select a given parameter $\delta \in(0,1)$. We say that $S_{\ell}$ is $\delta$-consistent whenever for each $p_{\ell} \in S_{\ell}$ it holds that $\delta p_{\ell} \in S_{\ell}$. Note that when $S_{\ell}=[0, b], \delta$-consistency is satisfied for any $\delta$. Nevertheless, when prices are established in legal tenders -see the description of $S^{G}$ in Subsection 2.2- divisibility problems might sever the connection between the local provider's strategy spaces in the two games.

\section{Lemma 1: An Equivalence Result}

Assume $S_{i} \subseteq[0, b]$ for each provider $i$. Let $\widehat{\delta} \in(0,1)$ be a given parameter such that $S_{\ell}$ is $\widehat{\delta}$ consistent, and $\widehat{\alpha}=1 / \widehat{\delta}$. Then $\left(p_{\ell}^{*}, p_{\mathfrak{f}}^{*}\right)$ is an undominated Nash equilibrium for the program with bias $\Gamma^{\widehat{\delta}}$ if and only if $\left(\widehat{\delta} p_{\ell}^{*}, p_{\mathfrak{f}}^{*}\right)$ is an undominated Nash equilibrium for the program with subsidy $\Gamma^{\widehat{\alpha}}$.

Several remarks are in order. First, the equivalence between the two affirmative action programs does not restrict only to equilibrium prices. The arguments in our proof for Lemma 1 imply that equilibrium provision shares (and hence supplier diversity) as well as payoffs are equivalent.

Second, while the equivalence between the affirmative action programs is intuitive, there are intricacies that come from the fact that in our setting the strategy space of providers is bounded by $b$. This makes the introduction of the safeguard clause necessary. The following example provides an intuition for the equivalence result and illustrates the role of the safeguard clause.

Example 4 We compare the equilibria for the FPA with bias $\delta$ game $\Gamma^{F P \delta}=\left\{I, S^{G}, \pi, \varphi^{F P \delta}\right\}$ and the FPA with subsidy $\alpha$ game $\Gamma^{F P \alpha C}=\left\{I, S^{G}, \pi^{\alpha C}, \varphi^{F P}\right\}$, where $S^{G}$ is defined in Subsection 2.2. To highlight the role of the safeguard clause consider also the FPA with subsidy $\alpha$ but without safeguard clause. This is the game $\Gamma^{F P \alpha}=\left\{I, S^{G}, \pi^{\alpha}, \varphi^{F P}\right\}$, which differs from $\Gamma^{F P \alpha C}$ in that the profit function $\pi^{\alpha}$ is defined by (6). Consider the procurement problem $\left(b, c_{\ell}, c_{\mathfrak{f}}\right)=$ $(100,90,84)$. Let $\alpha=1 / \delta=5 / 3$.

Notice that in the FPA with bias $\delta$ game $\Gamma^{F P \delta}$ the local provider receives a large discount. As $\delta b=60$, any permissible price of the local provider outbids the foreign supplier. The equilibria are hence $P^{*}=\left(p_{\ell}^{*}, p_{\mathfrak{f}}^{*}\right)=(100, x)$, where $x \in[84.01, b] \cap S^{G}$. In the FPA with subsidy $\alpha$, however, the local provider receives a premium when he wins. This provides an incentive to maximize the mark-up, provided the price is low enough to outbid the foreign supplier. In the game $\Gamma^{F P \alpha}$ without safeguard clause the local provider can set his price relatively high and just undercut the rival, as these prices are not dominated. Thus, the equilibria are $\tilde{P}=$ $\left(\tilde{p}_{\ell}, \tilde{p}_{\mathfrak{f}}\right)=(84, x)$, with $x>84$. Note that $\alpha \tilde{p}_{\ell}=140>b$. This implies that in the game $\Gamma^{F P \alpha C}$ with safeguard clause this price does not qualify for the entire premium. Hence this price is dominated for the local provider. It is thus better to lower the price to $b / \alpha$ and the equilibria are $\widehat{P}=\left(\widehat{p}_{\ell}, \widehat{p}_{\mathfrak{f}}\right)=(60, x)$, with $x>60$. This example shows that for the equilibrium prices of the local provider to fulfil the relationship $p_{\ell}^{*}=\alpha \widehat{p}_{\ell}$ the safeguard clause needs to be imposed.

Third, the equivalence result is more complex than a simple change of variable. The reason is that the safeguard clause makes prices close to the budget constraint less profitable but does not 
rule them out directly. Ruling out such prices would imply that the two providers have different strategy spaces, which is unappealing from a normative point of view. The next example shows that without the restriction to undominated prices in equilibrium the local provider might choose a price close to the budget constraint but this can only happen if there is no supplier diversity.

Example 5 Suppose the buyer has a preference for dual sourcing unless one supplier outbids the rival and the winning bid is sufficiently smaller than the budget. Formally, for each agent $i$ and a given parameter $k \in(0,1)$

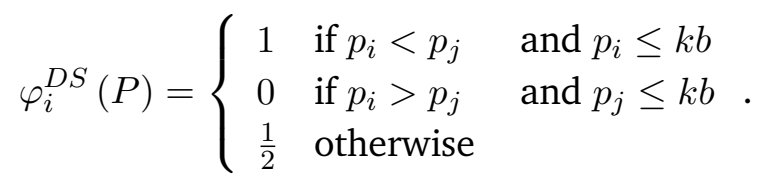

Using (15) rather than (2) in the games of Example 4 we obtain the program with bias $\delta$ game $\Gamma^{D S \delta}=\left\{I, S^{G}, \pi, \varphi^{D S \delta}\right\}$ and the program with subsidy $\alpha$ game $\Gamma^{D S \alpha C}=\left\{I, S^{G}, \pi^{\alpha C}, \varphi^{D S}\right\}$. We compare the equilibria for these games for the procurement problem $\left(b, c_{\ell}, c_{\mathfrak{f}}\right)=(100,88,50)$. Let $\alpha=1 / \delta=11 / 10$ and $k=0.8$. For conciseness of the exposition we focus on prices for which the mark-up of a supplier is strictly positive ${ }^{35}$

Consider the program with bias $\Gamma^{D S \delta}$. The affirmative action program is not very powerful and so the foreign supplier can outbid the local provider. This is because $\delta c_{\ell}=80$. The equilibria are hence $P^{*}=\left(p_{\ell}^{*}, p_{\mathfrak{f}}^{*}\right)=(x, 80)$, where $x \in[88.01, b] \cap S^{G}$. Notice that the specification of (15) implies that the foreign provider cannot raise his price without sharing the provision, which is unprofitable. Consider now the program with subsidy $\Gamma^{D S \alpha C}$. Because of the premium the local provider can lower his price until $\widehat{p}_{\ell}=80.01$, as $c_{\ell} / \alpha=80$. Thus, the equilibria are $\widehat{P}=\left(\widehat{p}_{\ell}, \widehat{p}_{\mathfrak{f}}\right)=(y, 80)$, where $y \in[80.01, b] \cap S^{G}$. Note that for each $y \in[80.01,90.90]$ that is part of an equilibrium for a program with subsidy $\alpha$ there is an $x=\alpha y$ that is part of an equilibrium for a program with bias $\delta$. For $y \in[90.91, b]$, however, this is not true, as $\alpha y>b$. These prices are only profitable, because there is no supplier diversity ${ }^{36}$

\subsection{Private Information}

The benchmark model of Section 3 considers the case in which providers are completely informed about each others' characteristics. While this is appropriate in situations in which the providers know each other well (Moldovanu and Sela, 2003; Bernheim and Whinston, 1986;

\footnotetext{
${ }^{35}$ For any given equilibrium, there might be another equilibrium in which one or both suppliers ask for one cent less than in the initial equilibrium.

${ }^{36}$ Notice that the statement of the relationship $x=\alpha y$ in this example and the analogue in Lemma 1 abstract from issues of divisibility when there is a finite grid on the strategy space. For instance, if $y=80.01$ then $\alpha y=88.011 \notin S^{G}$. In such a case there is an element in $S^{G}$ "close to $\alpha y$ " which is part of an equilibrium (in this example 88.01). Moreover, for $y \in[80.01,90.90]$ the difference between $\alpha y$ and the closest element in $S^{G}$ becomes smaller as the grid becomes finer. This is not true for $y \in[90.91, b]$. For example, if $y=91$, then $\alpha y=100.1>b$ and the distance to the closest element in $S^{G}$ is 0.1 , no matter how fine the grid.
} 
Anton and Yao, 1992), in other situations it is clearly unrealistic. Following Milgrom and Weber (1982), Edelman et al. (2007), and Alcalde and Dahm (2013, 2019) this assumption can be dispensed with. In fact, consider the other polar case in which each supplier only has (private) information about his own costs, but does not know the costs of his rival. Consider a variant of a reverse English (or Japanese) auction in which the buyer decreases the price continuously over time. Providers decide at what price to drop out. These drop out decisions are observed by the rival.

Even though providers initially do not have information about each other, during the course of the auction all the relevant information is revealed so that in equilibrium each supplier obtains the same share and payoffs as under complete information. The argument for this is similar to Alcalde and Dahm (2013, 2019)'s setting without affirmative action. In that setting it is key that the supplier dropping out first is certain to submit the higher price, and that the optimal higher price does not depend on the lowest price. Once the high-cost provider drops out, this drop out decision is observed by the low-cost supplier who resolves the trade-off between procurement share and mark-up optimally. This argument is unaffected by the introduction of affirmative action, because Theorem 1 establishes that whatever the intensity of the program the optimal higher price does not depend on the lower price.

\subsection{Multiple Sourcing}

Our model can be extended to more than two providers. One possibility to generalize it to $n$ providers is to use the recursive formulation in expression (1) in Alcalde and Dahm (2019).

To fix ideas consider a low-cost, an intermediate-cost, and a high-cost provider. In other words, in what follows we focus on three provider procurement problems $\left(b, c_{3}, c_{2}, c_{1}\right)$ with $0 \leq c_{1}<c_{2}<c_{3}<b$. Given a vector of prices such that $p_{3}>p_{2}>p_{1}$, the shares of the supply contract are

$$
\varphi_{3}^{C P}=\frac{b-p_{3}}{3\left(b-p_{1}\right)}, \quad \varphi_{2}^{C P}=\varphi_{3}^{C P}+\frac{p_{3}-p_{2}}{2\left(b-p_{1}\right)} \quad \text { and } \quad \varphi_{1}^{C P}=\varphi_{2}^{C P}+\frac{p_{2}-p_{1}}{b-p_{1}} .
$$

Assume that only the high-cost supplier 3 is targeted by affirmative action. Suppose that the intensity of affirmative action is low enough that, on one hand, $\alpha \leq c_{3} / c_{2}$ and, on the other hand, the safeguard clause does not apply, that is, $\alpha<\left(2 b-c_{3}\right) / b$. It can be shown that under these conditions Proposition 2 in Alcalde and Dahm (2019) applies. Similar to Theorem 1, this proposition guarantees the existence of an equilibrium in which providers behave as if the highcost supplier's cost were $c_{3} / \alpha$ instead of $c_{3}$ and the providers' equilibrium prices are ordered by their costs. In this equilibrium the high-cost provider 3 chooses the same price and the low-cost provider 1's price has a similar structure as in our benchmark model ${ }^{37}$ The intermediate-cost supplier 2's price has a similar structure to $p_{3}$ but also includes an adjustment to make it the

\footnotetext{
${ }^{37}$ For $p_{1}$, see expression 21 , in Appendix A.1.
} 
more competitive the higher the intensity of affirmative action. More precisely, we have that

$$
\begin{aligned}
& p_{3}=\frac{b+c_{3} / \alpha}{2}, \quad p_{2}=\frac{b+c_{2}}{2}-\frac{b-c_{3} / \alpha}{12} \text { and } \\
& p_{1}=b-\sqrt{\frac{\left(4 b-p_{3}-3 p_{2}\right)\left(b-c_{1}\right)}{6}} .
\end{aligned}
$$

To see that with more than three providers the main forces of our model remain intact and affirmative action still has the potential to help the local provider and induce more competitive procurement, consider the following example.

Example 6 Consider the following three procurement problems $\left(b, c_{3}, c_{2}, c_{1}\right)=(100,90,85,40)$, $\left(b, c_{3}, c_{2}, \hat{c}_{1}\right)=(100,90,85,36)$ and $\left(b, c_{3}, c_{2}, \tilde{c}_{1}\right)=(100,90,85,32)$. Notice that these problems differ only in the cost of the low-cost provider. The following table indicates for each problem and for three different intensities of affirmative action equilibrium prices and procurement expenditure.

\begin{tabular}{|cccc|c|ccc|c|}
\hline$b$ & $c_{3}$ & $c_{2}$ & $c_{1}$ & $\alpha$ & $p_{3}$ & $p_{2}$ & $p_{1}$ & $C(\alpha)$ \\
\hline 100 & 90 & 85 & 40 & 1.000 & 95.000 & 91.667 & 82.679 & 85.595 \\
100 & 90 & 85 & 40 & 1.025 & 93.902 & 91.484 & 82.211 & 85.505 \\
100 & 90 & 85 & 40 & 1.050 & 92.857 & 91.310 & 81.775 & 85.480 \\
\hline \hline 100 & 90 & 85 & 36 & 1.000 & 95.000 & 91.667 & 82.111 & 85.093 \\
100 & 90 & 85 & 36 & 1.025 & 93.902 & 91.484 & 81.627 & 84.984 \\
100 & 90 & 85 & 36 & 1.050 & 92.857 & 91.310 & 81.178 & 84.940 \\
\hline \hline 100 & 90 & 85 & 32 & 1.000 & 95.000 & 91.667 & 81.561 & 84.603 \\
100 & 90 & 85 & 32 & 1.025 & 93.902 & 91.484 & 81.062 & 84.476 \\
100 & 90 & 85 & 32 & 1.050 & 92.857 & 91.310 & 80.598 & 84.414 \\
\hline
\end{tabular}

In the first problem with $c_{1}=40$, the introduction of affirmative action reduces expenditure, but it does not seem to decrease it to a value below $c_{2}$. Decreasing the cost of the low-cost provider to $\hat{c}_{1}=36$ in the second problem makes affirmative action more beneficial. Affirmative action with intensity $2.5 \%$ reduces expenditure below $c_{2}$, while when there is no affirmative action -i.e., $\alpha=1$ - the equilibrium cost exceeds $c_{2}$. Lastly, decreasing the cost of the low-cost provider further to $\tilde{c}_{1}=32$ in the third problem yields a situation in which affirmative action is not needed to have lower expenditure than $c_{2}$. Nevertheless, such a policy (at intensity $2.5 \%$ or $5 \%$ ) reduces total cost further.

While this example suggests that our analysis can in principle be extended to multiple sourcing, it must be noted that in some circumstances the equilibrium is not unique ${ }^{38}$ One possible way to deal with this might be to apply a refinement to obtain uniqueness. Another possibility might be to use a different functional form to assign shares. In any case, a systematic analysis

\footnotetext{
${ }^{38}$ See the discussion in Alcalde and Dahm (2019).
} 
establishing a condition on the configuration of costs under which affirmative action is beneficial and varying the number of providers that can be targeted by affirmative action would be very interesting but is outside the scope of the present paper. We leave such an analysis for future research.

\section{Supplier Diversity before the Time of Cholera}

In this section we illustrate that endogenous set-asides are a better resilience measure than sole sourcing to mitigate shortages of, say, health products after a shock. We will also see that, since our approach gives the high-cost supplier a large share of the supply contract when the intensity of affirmative action is chosen optimally (Theorem 3), it performs better than other dual sourcing strategies that rely on less diversification of suppliers. To organise our discussion it is useful to distinguish between product shortages that are caused by supply disruptions and shortages following unexpected demand 39

Consider the following (intertemporal) framework. At time $t=0$ the Local Health Authority (LHA hereafter) buys $\sigma_{\mathfrak{f}}$ units from the foreign supplier and $\sigma_{\ell}=1-\sigma_{\mathfrak{f}}$ units from the local provider. When the supply contracts have to be delivered, at time $t=1$, supply disruptions or unexpected demand might occur. In both cases the health crisis allows the LHA to force the local provider to increase its production level and seize this production. Nevertheless, feasibility reasons might impede that the local supplier produces (instantaneously) as much as needed. For instance, to mitigate the worldwide shortage of face masks during the COVID-19 outbreak the World Health Organization (WHO) called on industry and governments to increase manufacturing by $40 \% 40$ Let $\mu>1$ denote the maximal increment that the local supplier can instantaneously produce ${ }^{41}$ Note that in a health crisis, the cost effectiveness objective of the LHA becomes negligible compared to the (negative) consequences of shortage. Moreover, the success of the LHA's policy is inversely related to the level of shortage, because the LHA needs to buy the net shortage in the international market. Depending on the type of shock, however, this might be very expensive or even technically unfeasible.

We will consider the net shortage defined as the difference between demand and available supply at time $t=1$. Comparing the net shortage arising from endogenous set-asides with the net shortage under dual sourcing, we will show that the former is always smaller than the latter. This implies that shortages are less frequent and less severe with endogenous set-asides than with sole sourcing. Following GlaxoSmithKline plc (2018) we consider the following two

\footnotetext{
${ }^{39}$ This follows the leading pharmaceutical firm GlaxoSmithKline plc. that notes: "Product shortages can happen for a variety of reasons, including supply disruptions and unexpected demand." See GlaxoSmithKline plc (2018, p. 29), available on its webpage, accessed on March 29, 2020.

${ }^{40}$ See for example the document ' Shortage of personal protective equipment endangering health workers worldwide', accessed on March 30, 2020.

${ }^{41}$ For instance, assume that $\mu=1.4$. This implies that the local supplier is able to instantaneously increase production by $40 \%$. Note that it is unrealistic to assume that a (local) pharmaceutical firm producing 1000 vaccine doses is able to instantaneously increase production to 1.000 .000 doses. The parameter $\mu$ is exogenously determined according to technical specifications of the technology.
} 
scenarios.

\subsection{Supply Disruptions}

An example for supply disruptions is the shortage of seasonal flu vaccines in the U.S. in 2004. This shortage was originated because Chiron, one of two suppliers, failed to produce the expected half of the necessary flu vaccines. The New York Times reported, 42

The primary fault lies with Chiron, an American biotechnology company, based in California, that had planned to supply some 46 million to 48 million doses of vaccine to the United States from a plant in Liverpool it acquired last year.

... British regulators suspended the firm's flu vaccine license early this month because of unspecified failures to comply with good manufacturing practices, suggesting a more deep-seated problem.

Since the vaccine doses are produced in Liverpool, the U.S. authorities do not have control over this production. Let $\phi \in[0,1)$ measure the shortage of the foreign provider's supply. For a given demand of vaccine doses (that we continue to normalize to one), with an endogenous set-aside the net shortage is $1-\left(\phi \sigma_{\mathfrak{f}}+\mu \sigma_{\ell}\right)$, while under sole sourcing net shortage is $1-\phi{ }^{43}$ The former is always smaller than the latter, as $\phi<\mu$. Hence, while under sole sourcing any supply disruption induces a shortage, with an endogenous set-aside shortages are less severe and happen only if the shock is large. To illustrate this further note that when the intensity of affirmative action is chosen optimally (Theorem 3), we have that $\sigma_{\mathfrak{f}}=\sigma_{\ell}$. Since a shortage occurs only if $\phi \sigma_{\mathfrak{f}}+\mu \sigma_{\ell}<1$, we obtain $\mu+\phi<2$. Using the value of $\mu=1$.4, as suggested by the WHO for the case of face masks, implies that with endogenous set-asides a shortage can be avoided for values up to $\phi \leq 0.6$. Other dual sourcing strategies with lower supplier diversity, however, avoid only smaller shocks.

\subsection{Unexpected Demand}

Unexpected demand is an important consequence of the COVID-19 outbreak. To illustrate the shortages in many countries around the world caused by this shock, consider face masks. ${ }^{44}$ The shortage of face masks is at least in part considered to be due to the concentration of most of the producers in China ${ }_{45}^{45}$ Before the outbreak many countries had not considered the production of

\footnotetext{
${ }^{42}$ See the Opinion piece entitled An Influenza Vaccine Debacle, accessed on March 29, 2020.

${ }^{43}$ The net shortage under sole sourcing makes the realistic assumption that a local supplier cannot start production instantaneously if the supplier was not active before the shock.

${ }^{44}$ An open letter published in March 22, 2020 in The Sunday Times entitled "Without protection, NHS staff are cannon fodder," illustrates how, in Great Britain, medics and other NHS staff urged prime minister Boris Johnson to take "immediate" action to ensure they have protective masks, glasses, gloves, aprons and suits. Accessed on March 29, 2020.

${ }^{45}$ See eg. "The World Needs Masks. China Makes Them - But Has Been Hoarding Them, that appeared on March 13, 2020 in the New York Times; accessed on March 31, 2020.
} 
face masks (as well as other medical supplies) to be a National Security concern. For instance, in Spain there is only one firm, Nueva Sibol, that produces protection FFP3 face masks. Nueva Sibol is a small firm and since 2019 owned by the Italian firm Spasciani ${ }^{46}$ Moreover, in Europe there are only six countries in which such face masks are produced ${ }^{47}$ As mentioned before, the WHO called on industry and governments to increase manufacturing by $40 \%$ to mitigate the worldwide shortage. At the same time an important share of some medical supplies cannot be distributed because they have not been produced according to European Union and/or United States certifications. This is due to the emergence of new producers that do not hold the required certification and the delay of public administrations in issuing the certifications. As a consequence of this, some firms have been exercising their market power at a moment of inelastic worldwide demand 48

Unexpected demand shocks can be incorporated in our framework as follows. Assume that at $t=0$, when the supply contract is offered, there is some uncertainty about the true needs at $t=1$, say $\rho$. Thus the LHA behaves as if $\rho$ is a random variable with density function $f$ and expected value 49

$$
E(\rho)=\int_{0}^{\infty} \rho f(\rho) d \rho=1 .
$$

When at $t=1$ the true value of $\rho$, say $\hat{\rho}$, is realised, a shortage due to unexpected demand appears whenever $\widehat{\rho}>1$, as the initial supply contract stipulates $\sigma_{\mathfrak{f}}+\sigma_{\ell}=1$. Assuming that a LHA can seize a local firm's production, consider the following cases.

(1) Unexpected Local Demand. In this case there is no unexpected demand in the foreign country so there is no reason why the foreign supplier's production is seized by its country authorities. With an endogenous set-aside the net shortage is $\widehat{\rho}-\left(\mu_{\mathfrak{f}} \sigma_{\mathfrak{f}}+\mu_{\ell} \sigma_{\ell}\right)$, while under sole sourcing net shortage is $\widehat{\rho}-\mu_{\mathfrak{f}}$. The former is smaller than the latter if and only if $\mu_{\mathfrak{f}} \leq \mu_{\ell}$. In the realistic case, however, in which $\mu$ is a decreasing function of the share of the supply contract (rather than a fixed proportion) or there are capacity constraints, shortages are less frequent and less severe with endogenous set-asides than under sole sourcing.

(2) Unexpected Global Demand. In this case there is unexpected demand in the foreign country too, as in the case of the COVID-19 outbreak. Consider the best case for the LHA and assume that the foreign firm fulfils its share $\sigma_{\mathfrak{f}}$ of the supply contract but does not provide more than this level. With an endogenous set-aside the net shortage is $\widehat{\rho}-\left(\sigma_{\mathfrak{f}}+\mu_{\ell} \sigma_{\ell}\right)$, while under sole sourcing net shortage is $\widehat{\rho}-1$. The former is always smaller than the

\footnotetext{
${ }^{46}$ Nueva Sibol's number of employees decreased from 39 in 2008 to 22 in 2018. Its operating income also decreased from $4326684 €$ in 2008 to $3812161 €$ in 2018.

${ }^{47}$ This information is based on an article in ABC, a Spanish newspaper, accessed on March 29, 2020.

${ }^{48}$ According the Independent, "WHO accuses medical suppliers of selling face masks at six times average price amid global crisis." Accessed on March 29, 2020.

${ }^{49}$ The following Condition 18 is imposed, because as in the main text we normalize the supply contract
} to 1 . 
latter, as $\mu_{\ell}>1$. Hence, while under sole sourcing any unexpected global demand shock creates a shortage, with an endogenous set-aside shortages are less severe and happen only if the shock is large. For instance, consider again a value of $\mu=1.4$ and the optimal intensity of affirmative action (Theorem 3), which implies that $\sigma_{\mathfrak{f}}=\sigma_{\ell}$. In this case an endogenous set-aside absorbs unexpected global demand shocks of up to $20 \%$. As the COVID-19 outbreak has shown, this resilience is important, because after an unexpected global demand shock it is impossible to buy the net shortage in the international market.

\section{Concluding Remarks}

This paper has introduced affirmative action in Alcalde and Dahm (2013)'s Contested Procurement Auction. This yields endogenous set-asides, since shares of the supply contract are allocated depending on the prices of suppliers. Affirmative action strengthens the local provider and induces him to set a more competitive price. This in turn results in the foreign supplier setting a more competitive price than he otherwise would and has the potential to lead to very competitive procurement. Our main result has shown that when the cost difference between providers is sufficiently large, the conflicting aims of supplier diversity and cost effectiveness can be reconciled. To do so the buyer has to choose the intensity of affirmative action in such a way that it levels the playing field completely. In equilibrium prices and shares of both providers are equal, so that supplier diversity is maximal. Moreover, the supply contract is allocated in a cost effective way, as provision costs are lower than those arising from a standard first-price auction. We have also considered extensions of the benchmark model, including an equivalence result for different affirmative action programs and a version of the share auction that allows to consider providers with private information about their costs.

While our main result requires the cost difference between providers to be sufficiently large, the possibility to use affirmative action benefits the buyer. This is because the trade-off between supplier diversity and cost effectiveness disappears in less demanding circumstances than in our earlier paper in which the buyer could not use affirmative action (Alcalde and Dahm, 2013). Our condition can be reinterpreted as saying that the ratio of the efficiency gains from opening the local market to foreign competition (that is, the cost difference of providers) to the efficiency gains in the local environment (that is, the difference between the reserve price and the local provider's cost) must be high enough. The less competitive the local provider is, the higher is this ratio and (it might be argued) the more affirmative action 'is needed' to 'protect the local supplier.' Hence, the more one expects to see political demands for affirmative action. Because of the large cost difference between providers, however, these programs appear prima facie to be very costly for society. Interestingly, our main result applies perhaps to those circumstances in which affirmative action is most controversial and says that -contrary to common wisdomthese programs can be designed in such a way that they are not costly.

A nice property of our model -which contrasts with those of a standard first-price auction- is that the equilibrium market share and profits of the local provider are always positive and very sensitive to the intensity of the affirmative action program. This is important from a dynamic 
perspective, as it might allow to reduce the cost difference between providers over time, so that affirmative action becomes unnecessary. There are at least two channels for this. First, profits might be reinvested in a better technology. Second, the greater the local provider's share of the supply contract, the more intense his learning process. Further work tackling these dynamics involves challenging questions for future research. The recent COVID-19 outbreak has shown, however, that understanding how diverse and profitable supply chains can be developed is of crucial importance from a National Security perspective.

\section{References}

Albano, G.L., Calzolari, G., Dini, F., Iossa, E., Spagnolo, G., 2006. Procurement contracting strategies, in: Dimitri, N., Piga, G., Spagnolo, G. (Eds.), Handbook of Procurement. Cambridge University Press, Cambridge, UK, pp. 82-120.

Alcalde, J., Dahm, M., 2007. Tullock and Hirshleifer: A meeting of the minds. Review of Economic Design 11, 101-124.

Alcalde, J., Dahm, M., 2011. On the complete information first-price auction and its intuitive solution. International Game Theory Review 13, 353-361.

Alcalde, J., Dahm, M., 2013. Competition for procurement shares. Games and Economic Behavior 80, 193-208.

Alcalde, J., Dahm, M., 2019. Dual sourcing with price discovery. Games and Economic Behavior $115,225-246$.

Anton, J.J., Yao, D.A., 1989. Split awards, procurement, and innovation. RAND Journal of Economics 20, 538-552.

Anton, J.J., Yao, D.A., 1992. Coordination in split award auctions. The Quarterly Journal of Economics 107, 681-707.

Athey, S., Coey, D., Levin, J., 2013. Set-asides and subsidies in auctions. American Economic Journal: Microeconomics 5, 1-27.

Ayres, I., Cramton, P., 1996. Deficit reduction through diversity: How affirmative action at the FCC increased auction competition. Stanford Law Review 48, 761-815.

Bag, P.K., Li, J., 2014. Bid coordination in split-award procurement: The buyer need not know anything. Economics Letters 124, 143 - 146.

Baldwin, R., Tomiura, E., 2020. Thinking ahead about the trade impact of COVID-19, in: Baldwin, R., di Mauro, B.W. (Eds.), Economics in the Time of COVID-19. Centre for Economic Policy Research Press, London, UK, pp. 59-71. 
Bernheim, D., Whinston, M.D., 1986. Menu auctions, resource allocation, and economic influence. The Quarterly Journal of Economics 101, 1-32.

Brannman, L., Froeb, L.M., 2000. Mergers, cartels, set-asides, and bidding preferences in asymmetric oral auctions. The Review of Economics and Statistics 82, 283-290.

Carpineti, L., Piga, G., Zanza, M., 2006. The variety of procurement practice: Evidence from public procurement, in: Dimitri, N., Piga, G., Spagnolo, G. (Eds.), Handbook of Procurement. Cambridge University Press, Cambridge, UK, pp. 14-44.

Che, Y.K., Gale, I., 1997. Rent dissipation when rent seekers are budget constrained. Public Choice 92, 109-126.

Chowdhury, S.M., Esteve-González, P., Mukherjee, A., 2019. Heterogeneity, leveling the playing field, and affirmative action in contests. Mimeographed.

Corns, A., Schotter, A., 1999. Can affirmative action be cost effective? an experimental examination of price-preference auctions. American Economic Review 89, 291-305.

Denes, T.A., 1997. Do small business set-asides increase the cost of government contracting? Public Administration Review 57, 441-444.

Edelman, B., Ostrovsky, M., Schwarz, M., 2007. Internet advertising and the generalized secondprice auction: Selling billions of dollars worth of keywords. American Economic Review 97, 242-259.

Engel, A.R., Ganuza, J.J., Hauk, E., Wambach, A., 2006. Managing risky bids, in: Dimitri, N., Piga, G., Spagnolo, G. (Eds.), Handbook of Procurement. Cambridge University Press, Cambridge, UK, pp. 322-344.

Esteban, J.M., Ray, D., 1999. Conflict and distribution. Journal of Economic Theory 87, 379415.

Ewerhart, C., Fieseler, K., 2003. Procurement auctions and unit-price contracts. The RAND Journal of Economics 34, 569-581.

Flambard, V., Perrigne, I., 2006. Asymmetry in procurement auctions: Evidence from snow removal contracts. The Economic Journal 116, 1014-1036.

Franke, J., 2012. Affirmative action in contest games. European Journal of Political Economy 28, 105-118.

Franke, J., Kanzow, C., Leininger, W., Schwartz, A., 2013. Effort maximization in asymmetric contest games with heterogeneous contestants. Economic Theory 52, 589-630.

Franke, J., Kanzow, C., Leininger, W., Schwartz, A., 2014. Lottery versus all-pay auction contests: A revenue dominance theorem. Games and Economic Behavior 83, 116-126. 
Franke, J., Leininger, W., Wasser, C., 2018. Optimal favoritism in all-pay auctions and lottery contests. European Economic Review 104, 22-37.

GlaxoSmithKline plc, 2018. GSK Annual Report 2018.

Holzer, H., Neumark, D., 2000. Assessing affirmative action. Journal of Economic Literature 38, 483-568.

Hubbard, T.P., Paarsch, H.J., 2009. Investigating bid preferences at low-price, sealed-bid auctions with endogenous participation. International Journal of Industrial Organization 27, $1-14$.

Jehiel, P., Lamy, L., 2015. On discrimination in auctions with endogenous entry. The American Economic Review 105, 2595-2643.

Jehiel, P., Lamy, L., 2019. On the benefits of set-asides. Journal of the European Economic Association, forthcoming.

Katz, R., Singer, D.A., 2007. Health and security in foreign policy. Bulletin of the World Health Organization 85, $161-244$.

Krasnokutskaya, E., Seim, K., 2011. Bid preference programs and participation in highway procurement auctions. The American Economic Review 101, 2653-2686.

Lee, J.S., 2008. Favoritism in asymmetric procurement auctions. International Journal of Industrial Organization 26, 1407-1424.

Loertscher, S., Marx, L.M., 2017. Auctions with bid credits and resale. International Journal of Industrial Organization 55, 58-90.

Marion, J., 2007. Are bid preferences benign? the effect of small business subsidies in highway procurement auctions. Journal of Public Economics 91, 1591-1624.

Marion, J., 2009. How costly is affirmative action? Government contracting and California's Proposition 209. Review of Economics and Statistics 91, 503-522.

Maskin, E., Riley, J., 2000. Asymmetric auctions. Review of Economic Studies 67, 413-438.

McAfee, R., McMillan, J., 1989. Government procurement and international trade. Journal of International Economics 26, 291-308.

Milgrom, P.R., Weber, R.J., 1982. A theory of auctions and competitive bidding. Econometrica 50, 1089-1122.

Moldovanu, B., Sela, A., 2003. Patent licensing to Bertrand competitors. International Journal of Industrial Organization 21, 1-13. 
Mummalaneni, S., 2019. Affirmative action as a cost cutting tool in procurement markets. Mimeographed, University of Washington.

Myerson, R.B., 1981. Optimal auction design. Mathematics of Operations Research 6, 58-73.

Nakabayashi, J., 2013. Small business set-asides in procurement auctions: An empirical analysis. Journal of Public Economics 100, 28-44.

Perry, M.K., Sákovics, J., 2003. Auctions for split-award contracts. Journal of Industrial Economics 51, 215-242.

Qiao, Y., Thai, K.V., Cummings, G., 2009. State and local procurement preferences: A survey. Journal of Public Procurement 9, 371-410.

Rothkopf, M.H., Harstad, R.M., Fu, Y., 2003. Is subsidizing inefficient bidders actually costly? Management Science 49, 71-84.

Szidarovszky, F., Okuguchi, K., 1997. On the existence and uniqueness of pure Nash equilibrium in rent-seeking games. Games and Economic Behavior 18, 135-140.

Wilson, R., 1979. Auctions of shares. The Quarterly Journal of Economics 93, 675-689.

\section{A Appendix}

\section{A.1 Proof of Theorem 1}

In this appendix we provide a formal proof of Theorem 1. To do so it is helpful to consider first the auxiliary game without safeguard clause. This is the game $\Gamma^{C P \alpha}=\left\{I, S, \pi^{\alpha}, \varphi^{C P}\right\}$, which differs from $\Gamma^{C P \alpha C}$ in that the profit function $\pi^{\alpha}$ is defined by (6). The next proposition characterizes the unique equilibrium of $\Gamma^{C P \alpha}$.

Proposition 3 The CPA with subsidy $\alpha>1$ but without safeguard clause has a unique equilibrium $\left(p_{\ell}(\alpha), p_{\mathfrak{f}}(\alpha)\right)$ described as follows.

(a) If $\alpha c_{f} \leq c_{\ell}$,

$$
\begin{aligned}
& p_{\ell}(\alpha)=\frac{\alpha b+c_{\ell}}{2 \alpha}, \text { and } \\
& p_{\mathfrak{f}}(\alpha)=b-\sqrt{\frac{\left(b-c_{\mathfrak{f}}\right)\left(\alpha b-c_{\ell}\right)}{4 \alpha}}
\end{aligned}
$$

(b) If $\alpha c_{f}>c_{\ell}$,

$$
\begin{aligned}
& p_{\ell}(\alpha)=b-\sqrt{\frac{\left(b-c_{\mathfrak{f}}\right)\left(\alpha b-c_{\ell}\right)}{4 \alpha}}, \text { and } \\
& p_{\mathfrak{f}}(\alpha)=\frac{b+c_{\mathfrak{f}}}{2}
\end{aligned}
$$




\section{Proof.}

Consider the CPA with subsidy $\alpha$ but without safeguard clause. Given the prices $\left(p_{\ell}, p_{\mathfrak{f}}\right)$ the profits of the local provider follow the expression

$$
\pi_{\ell}^{\alpha}\left(p_{\ell}, p_{\mathfrak{f}}\right)=\left\{\begin{array}{cc}
\frac{b-p_{\ell}}{2\left(b-p_{\mathfrak{f}}\right)}\left(\alpha p_{\ell}-c_{\ell}\right) & \text { if } p_{\ell} \geq p_{\mathfrak{f}} \\
{\left[1-\frac{b-p_{\mathfrak{f}}}{2\left(b-p_{\ell}\right)}\right]\left(\alpha p_{\ell}-c_{\ell}\right)} & \text { if } p_{\ell} \leq p_{\mathfrak{f}}
\end{array}\right.
$$

while the foreign provider's profits are

$$
\pi_{\mathfrak{f}}^{\alpha}\left(p_{\ell}, p_{\mathfrak{f}}\right)=\left\{\begin{array}{cl}
{\left[1-\frac{b-p_{\ell}}{2\left(b-p_{\mathfrak{f}}\right)}\right]\left(p_{\mathfrak{f}}-c_{\mathfrak{f}}\right)} & \text { if } p_{\ell} \geq p_{\mathfrak{f}} \\
\frac{b-p_{\mathfrak{f}}}{2\left(b-p_{\ell}\right)}\left(p_{\mathfrak{f}}-c_{\mathfrak{f}}\right) & \text { if } p_{\ell} \leq p_{\mathfrak{f}}
\end{array}\right.
$$

Note that equation 20) can be expressed as $\pi_{\mathfrak{f}}^{\alpha}\left(p_{\ell}, p_{\mathfrak{f}}\right)=\varphi_{\mathfrak{f}}^{C P}\left(p_{\ell}, p_{\mathfrak{f}}\right)\left(p_{\mathfrak{f}}-c_{\mathfrak{f}}\right)$, whereas equation (19) can be rewritten as

$$
\pi_{\ell}^{\alpha}\left(p_{\ell}, p_{\mathfrak{f}}\right)=\varphi_{\ell}^{C P}\left(p_{\ell}, p_{\mathfrak{f}}\right)\left(\alpha p_{\ell}-c_{\ell}\right)=\alpha \varphi_{\ell}^{C P}\left(p_{\ell}, p_{\mathfrak{f}}\right)\left(p_{\ell}-\frac{c_{\ell}}{\alpha}\right) .
$$

Consider a given problem with budget constraint $b$ and providers' cost $\left(c_{\ell}, c_{\mathfrak{f}}\right)$. The relationship described above implies that a vector of prices $\left(p_{\ell}^{*}, p_{\mathfrak{f}}^{*}\right)$ is an equilibrium for the problem without safeguard clause $\Gamma^{C P \alpha}$ if and only if $\left(p_{\ell}^{*}, p_{\mathfrak{f}}^{*}\right)$ is an equilibrium when there is no affirmative action, the budget constraint is $b$, and the providers' cost are $\left(c_{\ell} / \alpha, c_{\mathfrak{f}}\right)$. Note that the latter situation constitutes the original CPA, implying that the result follows from Corollary 1 in Alcalde and Dahm (2013).

In the proposition the threshold for the intensity of the affirmative action program $\widehat{\alpha}=c_{\ell} / c_{\mathfrak{f}}$ appears. For intensities lower than this threshold, the subsidy levels the playing field but does not change the behaviour in the sense that the local provider acts as high-cost supplier and the foreign provider undercuts his rival's price optimally. Once the intensity of the subsidy exceeds this threshold, however, behaviour is changed. The foreign provider behaves as a high-cost provider and local provider acts as low-cost supplier.

Equation (20) shows that, for $p_{\ell}$ given, the optimal decision by the foreign provider does not directly depend on the intensity parameter $\alpha$. Instead, it is (indirectly) affected by $\alpha$ through the local provider's price $p_{\ell}$. Simple optimization techniques allow to derive the foreign provider's reaction function $\sqrt[50]{ }$ It follows the expression

$$
R_{\mathfrak{f}}\left(p_{\ell}\right)=\left\{\begin{array}{cl}
\frac{b+c_{\mathfrak{f}}}{2} & \text { if } p_{\ell} \leq \frac{b+c_{\mathfrak{f}}}{2} \\
b-\sqrt{\frac{\left(b-c_{\mathfrak{f}}\right)\left(b-p_{\ell}\right)}{2}} & \text { if } p_{\ell} \geq \frac{b+c_{\mathfrak{f}}}{2}
\end{array}\right.
$$

\footnotetext{
${ }^{50}$ This function associates to each strategy selected by the local provider the optimal strategy of the foreign provider.
} 
Note that for $p_{\ell}$ small, the optimal decision by the foreign provider does not vary with $p_{\ell}$, while for $p_{\ell}$ high, this optimal decision increases with $p_{\ell}$.

Consider a given problem, with budget constraint $b$, and providers' costs $\left(c_{\ell}, c_{\mathfrak{f}}\right)$. Define the function $p_{\ell}:[1,+\infty) \rightarrow[0, b]$ as follows.

$$
p_{\ell}(\alpha)=\left\{\begin{array}{cc}
\frac{\alpha b+c_{\ell}}{2 \alpha} & \text { if } \alpha c_{\mathfrak{f}} \leq c_{\ell} \\
b-\sqrt{\frac{\left(b-c_{\mathfrak{f}}\right)\left(\alpha b-c_{\ell}\right)}{4 \alpha}} & \text { if } \alpha c_{\mathfrak{f}}>c_{\ell}
\end{array}\right.
$$

This function describes how the local provider's equilibrium price in Proposition 3 varies with $\alpha$. We have the following result.

Proposition 4 There is $\alpha^{*}$ such that $\alpha p_{\ell}(\alpha)<b$ if and only if $\alpha<\alpha^{*}$.

\section{Proof.}

First, observe that $p_{\ell}(\cdot)$ is a continuous function. Construct the function $\Delta:[1,+\infty) \rightarrow \mathbb{R}$ defined as $\Delta(\alpha)=b-\alpha p_{\ell}(\alpha)$. Note that $\Delta$ is also a continuous function. Moreover, since $b>c_{\ell}>c_{\mathfrak{f}}, \Delta(1)>0$. Consider the following two cases.

(a) $c_{\mathfrak{f}}=0$. Then, $\Delta$ is strictly decreasing in $[0,+\infty)$, and its unique root is

$$
\alpha^{*}=\frac{2 b-c_{\ell}}{b} .
$$

This implies that $\Delta(\alpha)>0$ if and only if $\alpha<\alpha^{*}$, as established in Proposition 4 ,

(b) $c_{\mathfrak{f}}>0$. Note that, since $\lim _{\alpha \rightarrow+\infty} \Delta(\alpha)=-\infty$, the continuity of $\Delta$ implies that there should be some $\alpha^{\prime}$ such that $\Delta\left(\alpha^{\prime}\right)<0$. Then, since $\Delta(1)>0$, Bolzano's Theorem guarantees the existence of $\alpha^{*}$ such that $\Delta\left(\alpha^{*}\right)=0$. To show that such a value for the intensity parameter is unique, note that

$$
\frac{\partial \Delta}{\partial \alpha}(\alpha)=\left\{\begin{array}{cl}
-\frac{b}{2} & \text { if } \alpha c_{\mathfrak{f}}<c_{\ell} \\
\frac{2 \alpha b-c_{\ell}}{4} \sqrt{\frac{b-c_{\mathfrak{f}}}{\alpha\left(\alpha b-c_{\ell}\right)}}-b & \text { if } \alpha c_{\mathfrak{f}}>c_{\ell}
\end{array}\right.
$$

Therefore, for $\alpha c_{f}>c_{\ell}$,

$$
b-c_{\mathfrak{f}}<\frac{\alpha b-c_{\ell}}{\alpha}
$$

and hence,

$$
\left.\frac{\partial \Delta}{\partial \alpha}\right|_{\alpha c_{\mathfrak{f}}>c_{\ell}}<\frac{2 \alpha b-c_{\ell}}{4 \alpha}-b=-\frac{2 b \alpha+c_{\ell}}{4 \alpha}<0 .
$$

Then, $\Delta$ is strictly decreasing, and thus its root is unique. 
Note that $\alpha^{*}$ is the minimal intensity level for which the safeguard clause becomes an effective constraint.

We are now ready to study the equilibria in the original game with safeguard clause $\Gamma^{C P \alpha C}$. Note that simple optimization techniques help to construct an equilibrium taking our Proposition 3 as a starting point. For $\alpha$ given, if $p_{\ell}(\alpha)$, as described by equation $(22)$, satisfies that $\alpha p_{\ell}(\alpha) \leq$ $b$, then the equilibrium described in Proposition 3 is still an equilibrium when the safeguard clause applies. Otherwise, an equilibrium is described as $p_{\ell}^{*}=b / \alpha$, while $p_{\mathfrak{f}}^{*}$ is obtained from equation (21) by taking $p_{\mathfrak{f}}^{*}=R_{\mathfrak{f}}(b / \alpha)$. Theorem 1 provides a more informative description of the equilibrium, since it explicitly states how the prices depend on the relevant parameters, that is, the budget constraint, the providers' costs and the intensity of the affirmative action program. We consider now the two scenarios distinguished in Theorem 1 . Notice that $c_{\ell} \geq 2 b c_{\mathfrak{f}} /\left(b+c_{\mathfrak{f}}\right)$ if and only if $b\left(c_{\ell}-c_{\mathfrak{f}}\right) \geq c_{\mathfrak{f}}\left(b-c_{\ell}\right)$.

(a) The cost difference of providers is large, that is, $b\left(c_{\ell}-c_{\mathfrak{f}}\right) \geq c_{\mathfrak{f}}\left(b-c_{\ell}\right)$. Define

$$
\alpha_{H}^{*}=\frac{2 b-c_{\ell}}{b} .
$$

Observe that

$$
\alpha_{H}^{*} c_{\mathfrak{f}} \leq c_{\ell} \Longleftrightarrow\left(2 b-c_{\ell}\right) c_{\mathfrak{f}} \leq c_{\ell} b \Longleftrightarrow\left(b-c_{\ell}\right) c_{\mathfrak{f}} \leq\left(c_{\ell}-c_{\mathfrak{f}}\right) b .
$$

This implies that, for intensity $\alpha<\alpha_{H}^{*}$, the safeguard clause does not impose an effective constraint. This is because the unrestricted equilibrium, described in Proposition 3 , satisfies that $\alpha p_{\ell}(\alpha) \leq b$.

Therefore, by Proposition 3 , we have that when $\alpha<\alpha_{H}^{*}$, the prices $\left(p_{\ell}^{*}, p_{\mathfrak{f}}^{*}\right)$ are an equilibrium if and only if

$$
\left(p_{\ell}^{*}, p_{\mathfrak{f}}^{*}\right)=\left(\frac{\alpha b+c_{\ell}}{2 \alpha}, b-\sqrt{\frac{\left(b-c_{\mathfrak{f}}\right)\left(\alpha b-c_{\ell}\right)}{4 \alpha}}\right) .
$$

Taking into account Proposition 4 we have that it is optimal for the local provider to select $p_{\ell}^{*}=b / \alpha$. Therefore, by equation (21), we have that for $\alpha>\alpha_{H}^{*},\left(p_{\ell}^{*}, p_{\mathfrak{f}}^{*}\right)$ is an equilibrium if and only if $p_{\ell}^{*}=b / \alpha$ and

$$
p_{\mathfrak{f}}^{*}=\left\{\begin{array}{cc}
\frac{b+c_{\mathfrak{f}}}{2} & \text { if } \alpha \geq \frac{2 b}{b+c_{\mathfrak{f}}} \\
b-\sqrt{\frac{(\alpha-1) b\left(b-c_{\mathfrak{f}}\right)}{2 \alpha}} & \text { if } \alpha<\frac{2 b}{b+c_{\mathfrak{f}}}
\end{array}\right.
$$

(b) The cost difference of providers is small, that is, $\left(c_{\ell}-c_{\mathfrak{f}}\right) b<\left(b-c_{\ell}\right) c_{\mathfrak{f}}$.

Consider the equilibrium function described in equation 22 . By equation 26 we have that the unique value $\alpha_{L}^{*}$ such that $\alpha_{L}^{*} p_{\ell}\left(\alpha_{L}^{*}\right)=b$ must satisfy that $c_{\ell}<\alpha_{L}^{*} c_{\mathfrak{f}}$. Therefore, by Proposition 4 we have that for each $\alpha$ such that $c_{\ell}>\alpha c_{\mathfrak{f}},\left(p_{\ell}^{*}, p_{\mathfrak{f}}^{*}\right)$ is an equilibrium if and only if it satisfies equation (27) above. 
Now, taking into account that, whenever $p_{\ell} \leq p_{\mathfrak{f}}$, the foreign provider' best response does not depend on the local provider's price -see equation (21) - it follows that, when $c_{\ell}<\alpha c_{\mathfrak{f}}$, the prices $\left(p_{\ell}^{*}, p_{\mathfrak{f}}^{*}\right)$ are an equilibrium if and only if $p_{\mathfrak{f}}^{*}=\left(b+c_{\mathfrak{f}}\right) / 2$ and

$$
p_{\ell}^{*}=\left\{\begin{array}{cl}
b-\sqrt{\frac{\left(b-c_{\mathfrak{f}}\right)\left(\alpha b-c_{\ell}\right)}{4 \alpha}} & \text { if } \alpha_{L}^{*} \geq \alpha>\frac{c_{\ell}}{c_{\mathfrak{f}}} \\
\frac{b}{\alpha} & \text { if } \alpha>\alpha_{L}^{*}
\end{array}\right.
$$

We observe that by Proposition $4, \alpha_{L}^{*}$ is the unique solution to

$$
2(\alpha-1) b=\sqrt{\alpha\left(b-c_{\mathfrak{f}}\right)\left(\alpha b-c_{\ell}\right)}
$$

given by

$$
\alpha_{L}^{*}=\frac{\left(8 b-c_{\ell}\right) b+c_{\ell} c_{\mathfrak{f}}+\sqrt{\left[16 b^{2}\left(b-c_{\ell}\right)+\left(b-c_{\mathfrak{f}}\right) c_{\ell}^{2}\right]\left(b-c_{\mathfrak{f}}\right)}}{2 b\left(3 b+c_{\mathfrak{f}}\right)} .
$$

We conclude this proof by defining six intervals for the intensity $\alpha$ of affirmative action. These intervals are based on the cases in Theorem 1 but for later reference we define them as open intervals. The definition of these intervals depends, on one hand, on the magnitude of the cost difference of providers and, on the other hand, on whether the intensity of affirmative action is low, intermediate or high. If the cost difference of providers is large, that is, $c_{\ell} \geq H\left(b, c_{\mathfrak{f}}\right)$, then we define low, intermediate and high intensity of affirmative action as $A_{l l}=\left(1, \alpha_{H}^{*}\right)$, $A_{l i}=\left(\alpha_{H}^{*}, \alpha^{e}\right)$, and $A_{l h}=\left(\alpha^{e}, \infty\right)$, respectively. This is illustrated in Figure 2 by the first procurement problem from Example 1, where we have that $A_{l l}=\left(1, \alpha_{1}^{*}\right), A_{l i}=\left(\alpha_{1}^{*}, \alpha_{1}^{e}\right)$, and $A_{l h}=\left(\alpha_{1}^{e}, \infty\right)$. If, however, $c_{\ell}<H\left(b, c_{\mathfrak{f}}\right)$, that is the cost difference of providers is small, then we define low, intermediate and high intensity of affirmative action as $A_{s l}=\left(1, \alpha^{e}\right), A_{s i}=\left(\alpha^{e}, \alpha_{L}^{*}\right)$, and $A_{s h}=\left(\alpha_{L}^{*}, \infty\right)$, respectively. This is illustrated in Figure 2 by the third procurement problem from Example 1. In this example we have that $A_{s l}=\left(1, \alpha_{3}^{e}\right), A_{s i}=\left(\alpha_{3}^{e}, \alpha_{3}^{*}\right)$, and $A_{s h}=\left(\alpha_{3}^{*}, \infty\right)$.

\section{A.2 Proof of Proposition 1}

Consider a procurement problem $\left(b, c_{\ell}, c_{\mathfrak{f}}\right)$ and assume that the intensity of the affirmative action policy is $\alpha>1$. For simplicity, given an affirmative action intensity $\alpha$, let $p_{\ell}^{e}(\alpha)$ denote the effective equilibrium price of the local provider; i.e., $p_{\ell}^{e}(\alpha)=\alpha p_{\ell}(\alpha)$. We show that the equilibrium provision share and revenue of the local (foreign) provider are increasing (decreasing, resp.) in the intensity of affirmative action. Since the extension to equilibrium profits is straightforward, we omit it here. It is useful to distinguish the six intervals for the intensity $\alpha$ of affirmative action policies defined at the end of the proof of Theorem 11. But since for policies with low and high intensities the equilibrium prices are independent of the cost difference of providers, in what follows we distinguish only three cases.

Case (a): Low intensity programs. 
Consider a procurement problem $\left(b, c_{\ell}, c_{\mathfrak{f}}\right)$ and assume that the intensity of the affirmative action policy is low, that is, it is either such that $\alpha \in A_{l l}=\left(1, \alpha_{H}^{*}\right)$ or $\alpha \in A_{s l}=\left(1, \alpha^{e}\right)$. In these cases-independent of the cost difference of providers-the safeguard clause is not binding and the local provider acts as the low-cost supplier. Note that the equilibrium prices are described in equation (27); that is

$$
\left(p_{\ell}^{e}(\alpha), p_{\mathfrak{f}}(\alpha)\right)=\left(\frac{\alpha b+c_{\ell}}{2}, b-\sqrt{\frac{\left(b-c_{\mathfrak{f}}\right)\left(\alpha b-c_{\ell}\right)}{4 \alpha}}\right) .
$$

This implies that,

$$
\frac{\partial p_{\ell}^{e}(\alpha)}{\partial \alpha}=\frac{b}{2}>0 \text {, while } \quad \frac{\partial p_{\mathfrak{f}}(\alpha)}{\partial \alpha}=-\frac{c_{\ell}}{4 \alpha^{2}} \sqrt{\frac{\alpha\left(b-c_{\mathfrak{f}}\right)}{\alpha b-c_{\ell}}}<0 .
$$

From equation $(32)$ also follows that

$$
\sigma_{\ell}(\alpha)=\sqrt{\frac{\alpha b-c_{\ell}}{4 \alpha\left(b-c_{\mathfrak{f}}\right)}} .
$$

From equation (34) above we have that

$$
\frac{\partial \sigma_{\ell}(\alpha)}{\partial \alpha}=\frac{c_{\ell}}{4 \alpha^{2}} \sqrt{\frac{\alpha}{\left(\alpha b-c_{\ell}\right)\left(b-c_{\mathfrak{f}}\right)}}>0 .
$$

Moreover, since for each $\alpha, \sigma_{\ell}(\alpha)+\sigma_{\mathfrak{f}}(\alpha) \equiv 1$, it follows that

$$
\frac{\partial \sigma_{\mathfrak{f}}(\alpha)}{\partial \alpha}=-\frac{\partial \sigma_{\ell}(\alpha)}{\partial \alpha}<0
$$

To conclude, note that the functions $p_{\ell}^{e}(\alpha), p_{\mathfrak{f}}(\alpha), \sigma_{\ell}(\alpha)$, and $\sigma_{\mathfrak{f}}(\alpha)$ are strictly positive, as well as continuously differentiable for any low intensity program. Then, by equations $(33)-(36)$,

$$
\begin{aligned}
\frac{\partial C_{\ell}(\alpha)}{\partial \alpha} & =\frac{\partial p_{\ell}^{e}(\alpha)}{\partial \alpha} \sigma_{\ell}(\alpha)+\frac{\partial \sigma_{\ell}(\alpha)}{\partial \alpha} p_{\ell}^{e}(\alpha)>0, \text { and } \\
\frac{\partial C_{\mathfrak{f}}(\alpha)}{\partial \alpha} & =\frac{\partial p_{\mathfrak{f}}(\alpha)}{\partial \alpha} \sigma_{\mathfrak{f}}(\alpha)+\frac{\partial \sigma_{\mathfrak{f}}(\alpha)}{\partial \alpha} p_{\mathfrak{f}}(\alpha)<0 .
\end{aligned}
$$

Case(b): Intermediate intensity programs. Here we distinguish two subcases.

Subcase (1): Intermediate intensity with small cost difference.

Consider a procurement problem $\left(b, c_{\ell}, c_{\mathfrak{f}}\right)$. Suppose that the cost difference of providers is small and that the intensity of the affirmative action policy is such that $\alpha \in A_{s i}=\left(\alpha^{e}, \alpha_{L}^{*}\right)$. In this case the intensity of the affirmative action program is high enough to induce the local provider to act as the low-cost supplier but it is not sufficient to make the safeguard clause binding. According to Theorem 1 , we have that 


$$
\left(p_{\ell}^{e}(\alpha), p_{\mathfrak{f}}(\alpha)\right)=\left(\alpha b-\sqrt{\frac{\left(\alpha b-c_{\ell}\right)\left(b-c_{\mathfrak{f}}\right) \alpha}{4}}, \frac{b+c_{\mathfrak{f}}}{2}\right) .
$$

Therefore,

$$
\frac{\partial p_{\ell}^{e}(\alpha)}{\partial \alpha}=b-\frac{2 \alpha b-c_{\ell}}{4} \sqrt{\frac{b-c_{\mathfrak{f}}}{\alpha\left(\alpha b-c_{\ell}\right)}} .
$$

Taking into account that $\alpha c_{\mathfrak{f}}>c_{\ell}$, from equation $(38)$ we have that

$$
\frac{\partial p_{\ell}^{e}(\alpha)}{\partial \alpha}>b-\frac{2 \alpha b-c_{\ell}}{4 \alpha}=\frac{b}{2}+\frac{c_{\ell}}{4 \alpha}>0 .
$$

Moreover, for the foreign provider we have that

$$
\frac{\partial p_{\mathfrak{f}}(\alpha)}{\partial \alpha}=0 .
$$

Given that $p_{\ell}(\alpha) \leq p_{\mathfrak{f}}(\alpha)$, the allocation for the local provider is

$$
\sigma_{\ell}(\alpha)=1-\sqrt{\frac{\alpha\left(b-c_{\mathfrak{f}}\right)}{4\left(\alpha b-c_{\ell}\right)}},
$$

and thus

$$
\frac{\partial \sigma_{\ell}(\alpha)}{\partial \alpha}=\frac{c_{\ell}}{4\left(\alpha b-c_{\ell}\right)^{2}} \sqrt{\frac{\left(\alpha b-c_{\ell}\right)\left(b-c_{\mathfrak{f}}\right)}{\alpha}}>0 .
$$

Therefore, taking into account that, for each $\alpha, \sigma_{\ell}(\alpha)+\sigma_{\mathfrak{f}}(\alpha) \equiv 1$, by equation 42 above we have that

$$
\frac{\partial \sigma_{\mathfrak{f}}(\alpha)}{\partial \alpha}=-\frac{\partial \sigma_{\ell}(\alpha)}{\partial \alpha}<0 .
$$

Finally, note that functions $p_{\ell}^{e}(\alpha), p_{\mathfrak{f}}(\alpha), \sigma_{\ell}(\alpha)$, and $\sigma_{\mathfrak{f}}(\alpha)$ are strictly positive, as well as continuously differentiable. Then, by equations (39)- 43,

$$
\begin{aligned}
& \frac{\partial C_{\ell}(\alpha)}{\partial \alpha}=\frac{\partial p_{\ell}^{e}(\alpha)}{\partial \alpha} \sigma_{\ell}(\alpha)+\frac{\partial \sigma_{\ell}(\alpha)}{\partial \alpha} p_{\ell}^{e}(\alpha)>0, \text { and } \\
& \frac{\partial C_{\mathfrak{f}}(\alpha)}{\partial \alpha}=\frac{\partial p_{\mathfrak{f}}(\alpha)}{\partial \alpha} \sigma_{\mathfrak{f}}(\alpha)+\frac{\partial \sigma_{\mathfrak{f}}(\alpha)}{\partial \alpha} p_{\mathfrak{f}}(\alpha)<0 .
\end{aligned}
$$

Subcase (2): Intermediate intensity programs with large cost difference.

Consider a procurement problem $\left(b, c_{\ell}, c_{\mathfrak{f}}\right)$. Suppose that the cost difference of providers is large and that the intensity of the affirmative action policy is such that $\alpha \in A_{l i}=\left(\alpha_{H}^{*}, \alpha^{e}\right)$. In 
this case the intensity of the affirmative action program is high enough to make the safeguard clause binding but it is not sufficient to induce the local provider to act as the low-cost supplier. According to Theorem 1 we have that, at the equilibrium,

$$
\left(p_{\ell}^{e}(\alpha), p_{\mathfrak{f}}(\alpha)\right)=\left(b, b-\sqrt{\frac{(\alpha-1) b\left(b-c_{\mathfrak{f}}\right)}{2 \alpha}}\right) .
$$

Therefore,

$$
\frac{\partial p_{\ell}^{e}(\alpha)}{\partial \alpha}=0 \text {, while } \frac{\partial p_{\mathfrak{f}}(\alpha)}{\partial \alpha}=-\frac{1}{2 \alpha^{2}} \sqrt{\frac{\left(b-c_{\mathfrak{f}}\right) \alpha b}{2(\alpha-1)}}<0 .
$$

Since $p_{\ell}(\alpha) \geq p_{\mathfrak{f}}(\alpha)$, we have that

$$
\sigma_{\ell}(\alpha)=\sqrt{\frac{(\alpha-1) b}{2\left(b-c_{\mathfrak{f}}\right) \alpha}}
$$

and thus

$$
\frac{\partial \sigma_{\ell}(\alpha)}{\partial \alpha}=\frac{1}{2 \alpha^{2}} \sqrt{\frac{\alpha b}{2(\alpha-1)\left(b-c_{\mathfrak{f}}\right)}}>0 .
$$

Note that, by equation (47), we can also derive that $\sigma_{\mathfrak{f}}(\cdot)$ is decreasing in the intensity level $\alpha$. Therefore,

$$
\begin{aligned}
\frac{\partial C_{\ell}(\alpha)}{\partial \alpha} & =\frac{\partial p_{\ell}^{e}(\alpha)}{\partial \alpha} \sigma_{\ell}(\alpha)+\frac{\partial \sigma_{\ell}(\alpha)}{\partial \alpha} p_{\ell}^{e}(\alpha)=\frac{\partial \sigma_{\ell}(\alpha)}{\partial \alpha} p_{\ell}^{e}(\alpha)>0, \text { while } \\
\frac{\partial C_{\mathfrak{f}}(\alpha)}{\partial \alpha} & =\frac{\partial p_{\mathfrak{f}}(\alpha)}{\partial \alpha} \sigma_{\mathfrak{f}}(\alpha)+\frac{\partial \sigma_{\mathfrak{f}}(\alpha)}{\partial \alpha} p_{\mathfrak{f}}(\alpha)<0 .
\end{aligned}
$$

\section{Case (c): High intensity programs.}

Consider a procurement problem $\left(b, c_{\ell}, c_{\mathfrak{f}}\right)$ and assume that the intensity of the affirmative action policy is high, that is, it is either such that $\alpha \in A_{l h}=\left(\alpha^{e}, \infty\right)$ or $\alpha \in A_{s h}=\left(\alpha_{L}^{*}, \infty\right)$. In these cases-independent of the cost difference of providers-the safeguard clause is binding and the local provider acts as the low-cost supplier. By Theorem 1 we have that, at the equilibrium,

$$
\left(p_{\ell}^{e}(\alpha), p_{\mathfrak{f}}(\alpha)\right)=\left(b, \frac{b+c_{\mathfrak{f}}}{2}\right) .
$$

Therefore,

$$
\frac{\partial p_{\ell}^{e}(\alpha)}{\partial \alpha}=\frac{\partial p_{\mathfrak{f}}(\alpha)}{\partial \alpha}=0 .
$$


Since $p_{\ell}(\alpha) \leq p_{\mathfrak{f}}(\alpha)$, we have that

$$
\sigma_{\mathfrak{f}}(\alpha)=\frac{\left(b-c_{\mathfrak{f}}\right) \alpha}{4(\alpha-1) b}
$$

and thus

$$
-\frac{\partial \sigma_{\ell}(\alpha)}{\partial \alpha}=\frac{\partial \sigma_{\mathfrak{f}}(\alpha)}{\partial \alpha}=-\frac{b-c_{\mathfrak{f}}}{4(\alpha-1)^{2} b}<0
$$

Therefore,

$$
\begin{aligned}
& \frac{\partial C_{\ell}(\alpha)}{\partial \alpha}=\frac{\partial p_{\ell}^{e}(\alpha)}{\partial \alpha} \sigma_{\ell}(\alpha)+\frac{\partial \sigma_{\ell}(\alpha)}{\partial \alpha} p_{\ell}^{e}(\alpha)=\frac{\partial \sigma_{\ell}(\alpha)}{\partial \alpha} p_{\ell}^{e}(\alpha)>0, \text { while } \\
& \frac{\partial C_{\mathfrak{f}}(\alpha)}{\partial \alpha}=\frac{\partial p_{\mathfrak{f}}(\alpha)}{\partial \alpha} \sigma_{\mathfrak{f}}(\alpha)+\frac{\partial \sigma_{\mathfrak{f}}(\alpha)}{\partial \alpha} p_{\mathfrak{f}}(\alpha)=\frac{\partial \sigma_{\mathfrak{f}}(\alpha)}{\partial \alpha} p_{\mathfrak{f}}(\alpha)<0 .
\end{aligned}
$$

\section{A.3 The Buyer's Total Provision Costs}

In this appendix we explore how the buyer's total provision costs vary when the intensity of affirmative action changes. Given a procurement problem $\left(b, c_{\ell}, c_{\mathfrak{f}}\right)$ and an affirmative action intensity $\alpha$, the total cost function $C(\alpha)$ is described by

$$
C(\alpha)=p_{\ell}^{e}(\alpha) \sigma_{\ell}(\alpha)+p_{\mathfrak{f}}(\alpha) \sigma_{\mathfrak{f}}(\alpha),
$$

where $\sigma_{i}(\alpha)$ and $p_{i}(\alpha)$ are the equilibrium allocation and the equilibrium price of provider $i$, while $p_{\ell}^{e}(\alpha)=\alpha p_{\ell}(\alpha)$ is the effective price of the local supplier.

Note that $C(\alpha)$ is a continuous function. Moreover, for $\alpha>1, C(\alpha)$ is continuously differentiable, except at (at most) two different values of $\alpha$. These values are $\alpha^{*}$ and $\alpha^{e}$. They delimit the intervals of intermediate affirmative action intensity, as introduced at the end of the proof of Theorem 1. The discussion in Appendix A.2 allows us to focus our analysis on the values of $\alpha$ for which the equilibrium prices $p_{\ell}(\cdot)$ and $p_{\mathfrak{f}}(\cdot)$, as well as the allocation functions $\sigma_{\ell}(\cdot)$ and $\sigma_{\mathfrak{f}}(\cdot)$ are continuously differentiable.

Taking into account that, by construction, $\sigma_{\ell}(\alpha)+\sigma_{\mathfrak{f}}(\alpha) \equiv 1$, for any $\alpha^{\prime}$-at which $C(\cdot)$ is differentiable- we have that

$$
\left.\begin{array}{rl}
\frac{\partial C\left(\alpha^{\prime}\right)}{\partial \alpha} & =\frac{\partial C_{\ell}\left(\alpha^{\prime}\right)}{\partial \alpha}+\frac{\partial C_{\mathfrak{f}}\left(\alpha^{\prime}\right)}{\partial \alpha} \\
& =\left(\frac{\partial p_{\ell}^{e}\left(\alpha^{\prime}\right)}{\partial \alpha}-\frac{\partial p_{\mathfrak{f}}\left(\alpha^{\prime}\right)}{\partial \alpha}\right) \sigma_{\ell}\left(\alpha^{\prime}\right)+\frac{\partial p_{\mathfrak{f}}\left(\alpha^{\prime}\right)}{\partial \alpha}+ \\
& +\left[p_{\ell}^{e}\left(\alpha^{\prime}\right)-p_{\mathfrak{f}}\left(\alpha^{\prime}\right)\right] \frac{\partial \sigma_{\ell}\left(\alpha^{\prime}\right)}{\partial \alpha} .
\end{array}\right\}
$$

\section{Proof of Proposition 2}

Using the notation introduced at the end of the proof of Theorem 1, we consider the following cases. 
(a) Large cost difference, that is, $c_{\ell} \geq H\left(b, c_{\mathfrak{f}}\right)$ and $\alpha \in A_{l h}=\left(\alpha^{e}, \infty\right)$.

Note that, by Theorem 1, for each $\alpha^{\prime}>\alpha^{e}, p_{\ell}^{e}\left(\alpha^{\prime}\right)=b$, while $p_{\mathfrak{f}}\left(\alpha^{\prime}\right)=\left(b+c_{\mathfrak{f}}\right) / 2$. This allows to simplify equation $(52)$ to

$$
\frac{\partial C\left(\alpha^{\prime}\right)}{\partial \alpha}=\left[p_{\ell}^{e}\left(\alpha^{\prime}\right)-p_{\mathfrak{f}}\left(\alpha^{\prime}\right)\right] \frac{\partial \sigma_{\ell}\left(\alpha^{\prime}\right)}{\partial \alpha} .
$$

Then, since $b>c_{\mathfrak{f}}$, and thus $p_{\ell}^{e}\left(\alpha^{\prime}\right)>p_{\mathfrak{f}}\left(\alpha^{\prime}\right)$, taking into account equation (51), it follows that $C$ is strictly increasing on $\alpha$ whenever $\alpha>\alpha^{e}$.

(b) Small cost difference, that is, $c_{\ell}<H\left(b, c_{\mathfrak{f}}\right)$ and $\alpha \in A_{s h}=\left(\alpha_{L}^{*}, \infty\right)$.

We distinguish two scenarios. First, consider the high intensity case: $\alpha^{\prime}>\alpha_{H}^{*} \geq \alpha^{e}$, where $\alpha_{H}^{*}$ is defined in equation (25). By Theorem 1 , for each such $\alpha^{\prime}, p_{\ell}^{e}\left(\alpha^{\prime}\right)=b$, while $p_{\mathfrak{f}}\left(\alpha^{\prime}\right)=\left(b+c_{\mathfrak{f}}\right) / 2$. Therefore, the arguments above are still valid to conclude that $C$ is strictly increasing.

Second, consider now the intermediate intensity case, where $\alpha^{e}<\alpha^{\prime}<\alpha_{H}^{*}$. Theorem 1 establishes that, at the equilibrium, the relevant prices are described as in equation (37), that is,

$$
\left(p_{\ell}^{e}\left(\alpha^{\prime}\right), p_{\mathfrak{f}}\left(\alpha^{\prime}\right)\right)=\left(\alpha^{\prime} b-\sqrt{\frac{\left(\alpha^{\prime} b-c_{\ell}\right)\left(b-c_{\mathfrak{f}}\right) \alpha^{\prime}}{4}}, \frac{b+c_{\mathfrak{f}}}{2}\right) .
$$

Let define $\Lambda(\alpha)=p_{\ell}^{e}(\alpha)-p_{\mathfrak{f}}(\alpha)$. Note that, for $\alpha^{e}<\alpha^{\prime}<\alpha_{H}^{*}$,

$$
\Lambda\left(\alpha^{\prime}\right)=\alpha^{\prime} b-\sqrt{\frac{\alpha^{\prime}\left(b-c_{\mathfrak{f}}\right)\left(\alpha^{\prime} b-c_{\ell}\right)}{4}}-\frac{b+c_{\mathfrak{f}}}{2} .
$$

Then, by equation $(39)$, we have that

$$
\frac{\partial \Lambda\left(\alpha^{\prime}\right)}{\partial \alpha}=\frac{\partial p_{\ell}^{e}\left(\alpha^{\prime}\right)}{\partial \alpha}>0
$$

Additionally, by equation (54), since $\alpha^{\prime}>\alpha^{e}$,

$$
\Lambda\left(\alpha^{\prime}\right)>\alpha^{\prime} b-\frac{\alpha^{\prime}\left(b-c_{\mathfrak{f}}\right)}{2}-\frac{b+c_{\mathfrak{f}}}{2}=\left(\alpha^{\prime}-1\right) \frac{b+c_{\mathfrak{f}}}{2}>0 .
$$

Recall that, by equation (42), $\sigma_{\ell}(\alpha)$ is an increasing function. Moreover, by construction, $0 \leq \sigma_{\ell}(\alpha) \leq 1$. Therefore, by equation $(52), C(\cdot)$ is an increasing function for each $\alpha \in\left(\alpha^{e}, \alpha_{H}^{*}\right)$.

\section{Proof of Theorem 2}

Theorem 2 can be proved by analysing the buyer's cost function when $\alpha$ approaches 1 (from above). Consider a procurement problem $\left(b, c_{\ell}, c_{\mathfrak{f}}\right)$ and assume that the intensity of the affirmative action policy is low. Using the notation defined at the end of the proof of Theorem 1 . 
suppose that either $\alpha \in A_{l l}=\left(1, \alpha_{H}^{*}\right)$ or $\alpha \in A_{s l}=\left(1, \alpha^{e}\right)$. Note that, since $b>c_{\ell}$ and $c_{\ell}>c_{\mathfrak{f}}$, both $A_{l l}$ and $A_{s l}$ are non-empty open intervals.

Moreover, by equation (32), for low intensity programs, the equilibrium prices are

$$
\left(p_{\ell}(\alpha), p_{\mathfrak{f}}(\alpha)\right)=\left(\frac{\alpha b+c_{\ell}}{2 \alpha}, b-\sqrt{\frac{\left(b-c_{\mathfrak{f}}\right)\left(\alpha b-c_{\ell}\right)}{4 \alpha}}\right) .
$$

Therefore, by equations 33 to 36 ,

$$
\lim _{\alpha^{\prime} \rightarrow 1^{+}} p_{\ell}^{e}\left(\alpha^{\prime}\right) \frac{\partial \sigma_{\ell}}{\partial \alpha}\left(\alpha^{\prime}\right)+\lim _{\alpha^{\prime} \rightarrow 1^{+}} p_{\mathfrak{f}}\left(\alpha^{\prime}\right) \frac{\partial \sigma_{\mathfrak{f}}}{\partial \alpha}\left(\alpha^{\prime}\right)=\frac{c_{\ell}}{8}\left(1-\sqrt{\frac{b-c_{\ell}}{b-c_{\mathfrak{f}}}}\right),
$$

and

$$
\lim _{\alpha^{\prime} \rightarrow 1^{+}}\left[\sigma_{\ell}\left(\alpha^{\prime}\right) \frac{\partial p_{\ell}^{e}}{\partial \alpha}\left(\alpha^{\prime}\right)+\sigma_{\mathfrak{f}}\left(\alpha^{\prime}\right) \frac{\partial p_{\mathfrak{f}}}{\partial \alpha}\left(\alpha^{\prime}\right)\right]=\frac{c_{\ell}}{8}+\frac{1}{4}\left(b \sqrt{\frac{b-c_{\ell}}{b-c_{\mathfrak{f}}}}-c_{\ell} \sqrt{\frac{b-c_{\mathfrak{f}}}{b-c_{\ell}}}\right) .
$$

Therefore,

$$
\lim _{\alpha^{\prime} \rightarrow 1^{+}} \frac{\partial C\left(\alpha^{\prime}\right)}{\partial \alpha}<0 \Longleftrightarrow \frac{c_{\ell}}{8}\left(1-\sqrt{\frac{b-c_{\ell}}{b-c_{\mathfrak{f}}}}\right)+\frac{c_{\ell}}{8}+\frac{1}{4}\left(b \sqrt{\frac{b-c_{\ell}}{b-c_{\mathfrak{f}}}}-c_{\ell} \sqrt{\frac{b-c_{\mathfrak{f}}}{b-c_{\ell}}}\right)<0 .
$$

Note that the last inequality can be rewritten as $\beta\left(b, c_{\ell}, c_{\mathfrak{f}}\right) / 4<0$. Therefore $\beta\left(b, c_{\ell}, c_{\mathfrak{f}}\right)<0$ is a necessary and sufficient condition to guarantee that the buyers' cost decreases with the intensity of affirmative action program when it is close to zero (i.e., $\alpha \rightarrow 1^{+}$).

\section{Proof of Theorem 3}

Assume that $c_{\ell} \geq H\left(b, c_{\mathfrak{f}}\right)$, and thus $\alpha^{*}=\left(2 b-c_{\ell}\right) / b$. We start by proving that provision costs are locally minimized at $\alpha^{e}$. More precisely, we will see that for $\alpha>\alpha^{*}, C(\alpha) \geq C\left(\alpha^{e}\right)$. Using the notation defined at the end of the proof of Theorem 1 , suppose that the intensity of the affirmative action program is either intermediate or high. Hence, either $\alpha \in A_{l i}=\left(\alpha^{*}, \alpha^{e}\right)$ or $\alpha \in A_{l h}=\left(\alpha^{e}, \infty\right)$. The expressions used throughout this proof (i.e., partial derivatives and/or limits) are computed under the assumption that the intensity belongs to the interval under consideration.

(1) High intensity affirmative action programs, that is, $\alpha>\alpha^{e}$.

By Proposition 2, for high intensity affirmative action programs, we have that

$$
\frac{\partial C(\alpha)}{\partial \alpha}>0
$$

Note that, when the high intensity $\alpha$ goes to $\alpha^{e}, p_{\ell}^{e}(\alpha)-p_{\mathfrak{f}}(\alpha)$ goes to $\left(b-c_{\mathfrak{f}}\right) / 2>0$. Additionally, since $\alpha^{e}=2 b /\left(b+c_{\mathfrak{f}}\right)>1$, by equation (51),

$$
\lim _{\alpha \rightarrow\left(\alpha^{e}\right)^{+}} \frac{\partial \sigma_{\ell}(\alpha)}{\partial \alpha}=\frac{b-c_{\mathfrak{f}}}{4\left(\alpha^{e}-1\right)^{2} b}>0 .
$$

(2) Intermediate intensity affirmative action programs, that is, $\alpha^{*}<\alpha<\alpha^{e}$. 
Note that, by equation (45), whenever $\alpha^{*}<\alpha<\alpha^{e}$,

$$
\frac{\partial C(\alpha)}{\partial \alpha}=\left(p_{\ell}^{e}(\alpha)-p_{\mathfrak{f}}(\alpha)\right) \frac{\partial \sigma_{\ell}(\alpha)}{\partial \alpha}+\frac{\partial p_{\mathfrak{f}}(\alpha)}{\partial \alpha} \sigma_{\mathfrak{f}}(\alpha) .
$$

Moreover, by equation (44),

$$
p_{\ell}^{e}(\alpha)-p_{\mathfrak{f}}(\alpha)=\sqrt{\frac{(\alpha-1) b\left(b-c_{\mathfrak{f}}\right)}{2 \alpha}} ;
$$

and thus, by equation 47 , it follows that

$$
\left(p_{\ell}^{e}(\alpha)-p_{\mathfrak{f}}(\alpha)\right) \frac{\partial \sigma_{\ell}(\alpha)}{\partial \alpha}=\frac{b}{4 \alpha^{2}} .
$$

From equations 45 to 47 we have that

$$
\frac{\partial p_{\mathfrak{f}}(\alpha)}{\partial \alpha} \sigma_{\mathfrak{f}}(\alpha)=\frac{1}{2 \alpha^{2}}\left[\frac{b}{2}-\sqrt{\frac{\left(b-c_{\mathfrak{f}}\right) \alpha b}{2(\alpha-1)}}\right] .
$$

Therefore, combining equations (60) to 63),

$$
\frac{\partial C(\alpha)}{\partial \alpha}=\frac{1}{2 \alpha^{2}}\left[b-\sqrt{\frac{\left(b-c_{\mathfrak{f}}\right) \alpha b}{2(\alpha-1)}}\right] .
$$

Taking into account that $\alpha<\alpha^{e}$, and thus $\alpha\left(b+c_{\mathfrak{f}}\right)<2 b$, it follows that

$$
\begin{aligned}
& {\left[\alpha\left(b+c_{\mathfrak{f}}\right)<2 b\right] \Leftrightarrow\left[\alpha c_{\mathfrak{f}}<(2-\alpha) b\right] \Leftrightarrow\left[2(\alpha-1) b<\alpha\left(b-c_{\mathfrak{f}}\right)\right] \Leftrightarrow} \\
& {\left[2(\alpha-1) b^{2}<\alpha b\left(b-c_{\mathfrak{f}}\right)\right] \Leftrightarrow\left[b<\sqrt{\frac{\alpha\left(b-c_{\mathfrak{f}}\right) b}{2(\alpha-1)}}\right] \Leftrightarrow \frac{\partial C(\alpha)}{\partial \alpha}<0 .}
\end{aligned}
$$

Moreover, from the above chain of equivalences we can also derive that

$$
\lim _{\alpha \rightarrow \alpha^{e}} \frac{\partial C(\alpha)}{\partial \alpha}=0 .
$$

This demonstrates that the provision cost reach a local minimum at $\alpha=\alpha^{e}$.

The remainder of this appendix is devoted to prove that when the cost difference is large enough the optimal intensity level of affirmative action is easily identifiable. The level of heterogeneity guaranteeing our result is established in Condition 1 below:

Condition 1 We assume that $0 \leq c_{\mathfrak{f}}<\left(3 b+c_{\mathfrak{f}}\right) / 4 \leq c_{\ell}<b$.

\footnotetext{
${ }^{51}$ Note that, since we assume that $0 \leq c_{\mathfrak{f}}<c_{\ell}<b$, Condition 1 is equivalent to the requirement that equation (11) in Theorem 3 is satisfied.
} 
Observe that, under Condition 1 , it holds that

$$
\frac{2 b-c_{\ell}}{b}-\frac{2 b}{b+c_{\mathfrak{f}}} \leq \frac{5 b-c_{\mathfrak{f}}}{4 b}-\frac{2 b}{b+c_{\mathfrak{f}}}=-\frac{3 b^{2}+c_{\mathfrak{f}}^{2}-4 b c_{\mathfrak{f}}^{2}}{4 b\left(b+c_{\mathfrak{f}}\right)}=-\frac{\left(3 b-c_{\mathfrak{f}}\right)\left(b-c_{\mathfrak{f}}\right)}{4 b\left(b+c_{\mathfrak{f}}\right)}<0,
$$

and thus

$$
\alpha^{*}=\sup \left\{\alpha \geq 1: \alpha p_{\ell}(\alpha)<b\right\}=\frac{2 b-c_{\ell}}{b}<\frac{2 b}{b+c_{\mathfrak{f}}}=\alpha^{e},
$$

where $\alpha^{e}$ is the unique intensity level $\alpha$ satisfying that $p_{\ell}(\alpha)=p_{\mathfrak{f}}(\alpha)$.

Recall that, for $b, c_{\ell}$ and $c_{\mathfrak{f}}$ given, the buyers' cost at the equilibrium, when an affirmative action program with intensity $\alpha$ is implemented, can be described as

$$
C(\alpha)=p_{\ell}^{e}(\alpha) \sigma_{\ell}(\alpha)+p_{\mathfrak{f}}(\alpha)\left(1-\sigma_{\ell}(\alpha)\right)=\left[p_{\ell}^{e}(\alpha)-p_{\mathfrak{f}}(\alpha)\right] \sigma_{\ell}(\alpha)+p_{\mathfrak{f}}(\alpha) .
$$

Moreover, when the parameters fulfill Condition 1, and $\alpha<\alpha^{*}$, it follows that

(a) The share for the local provider is

$$
\sigma_{\ell}(\alpha)=\sqrt{\frac{\alpha b-c_{\ell}}{4 \alpha\left(b-c_{\mathfrak{f}}\right)}} .
$$

(b) The effective price for the local provider is

$$
p_{\ell}^{e}(\alpha)=\frac{\alpha b+c_{\ell}}{2}
$$

(c) The price for the foreign provider is

$$
p_{\mathfrak{f}}(\alpha)=b-\sqrt{\frac{\left(b-c_{\mathfrak{f}}\right)\left(\alpha b-c_{\ell}\right)}{4 \alpha}} .
$$

To conclude the proof of Theorem 3 we just need to see that, for each $\alpha \in A_{l l}=\left[1, \alpha^{*}\right]$,

$$
C(\alpha) \geq \frac{3 b+c_{\mathfrak{f}}}{4}=C\left(\alpha^{e}\right) .
$$

By equation (66), this is equivalent to show that, for $\alpha \leq \alpha^{*}$,

$$
\mathcal{A}(\alpha)=p_{\ell}^{e}(\alpha)-p_{\mathfrak{f}}(\alpha)-\left[\frac{3 b+c_{\mathfrak{f}}}{4}-p_{\mathfrak{f}}(\alpha)\right] \sigma_{\ell}^{-1}(\alpha) \geq 0 .
$$

It is important to stress that, since $\alpha^{*} b=2 b-c_{\ell}$, whenever Condition 1 is fulfilled, for each $\alpha \leq \alpha^{*}$

$$
\alpha b-c_{\ell} \leq \frac{b-c_{\mathfrak{f}}}{2} .
$$


Taking into account the expressions of the auxiliary functions $p_{\ell}^{e}, p_{\mathfrak{f}}$ and $\sigma_{\ell}$ described above we have that

$$
\begin{aligned}
& \mathcal{A}(\alpha)=p_{\ell}^{e}(\alpha)-p_{\mathfrak{f}}(\alpha)-\left[\frac{3 b+c_{\mathfrak{f}}}{4}-p_{\mathfrak{f}}(\alpha)\right] \sigma_{\ell}^{-1}(\alpha) \\
& \frac{\alpha b+c_{\ell}}{2}-b+\sqrt{\frac{\left(b-c_{\mathfrak{f}}\right)\left(\alpha b-c_{\ell}\right)}{4 \alpha}}+\frac{b-c_{\mathfrak{f}}}{4} \sqrt{\frac{4 \alpha\left(b-c_{\mathfrak{f}}\right)}{\alpha b-c_{\ell}}}-\left(b-c_{\mathfrak{f}}\right) \\
& \frac{\alpha b-c_{\ell}}{2}-\left(b-c_{\ell}\right)-\left(b-c_{\mathfrak{f}}\right)+\sqrt{\frac{\left(b-c_{\mathfrak{f}}\right)\left(\alpha b-c_{\ell}\right)}{4 \alpha}}+\frac{b-c_{\mathfrak{f}}}{4} \sqrt{\frac{4 \alpha\left(b-c_{\mathfrak{f}}\right)}{\alpha b-c_{\ell}}}
\end{aligned}
$$

Note that, by Condition 1, $4\left(b-c_{\ell}\right) \leq b-c_{\mathfrak{f}}$, and thus

$$
\begin{aligned}
\mathcal{A}(\alpha) & \geq \frac{\alpha b-c_{\ell}}{\alpha\left(b-c_{\mathfrak{f}}\right)} \frac{\alpha\left(b-c_{\mathfrak{f}}\right)}{2}+\frac{b-c_{\mathfrak{f}}}{2}\left[\sqrt{\frac{\alpha\left(b-c_{\mathfrak{f}}\right)}{\alpha b-c_{\ell}}}+\sqrt{\frac{\alpha b-c_{\ell}}{\alpha\left(b-c_{\mathfrak{f}}\right)}}-\frac{5}{2}\right]= \\
& =\frac{b-c_{\mathfrak{f}}}{2}\left[\frac{\alpha b-c_{\ell}}{\alpha\left(b-c_{\mathfrak{f}}\right)} \alpha+\sqrt{\frac{\alpha\left(b-c_{\mathfrak{f}}\right)}{\alpha b-c_{\ell}}}+\sqrt{\frac{\alpha b-c_{\ell}}{\alpha\left(b-c_{\mathfrak{f}}\right)}}-\frac{5}{2}\right] .
\end{aligned}
$$

Since $\alpha \geq 1$,

$$
\mathcal{A}(\alpha) \geq \frac{b-c_{\mathfrak{f}}}{2}\left[\frac{\alpha b-c_{\ell}}{\alpha\left(b-c_{\mathfrak{f}}\right)}+\sqrt{\frac{\alpha\left(b-c_{\mathfrak{f}}\right)}{\alpha b-c_{\ell}}}+\sqrt{\frac{\alpha b-c_{\ell}}{\alpha\left(b-c_{\mathfrak{f}}\right)}}-\frac{5}{2}\right] .
$$

Consider the function $f(x)=x^{-1}+x^{1 / 2}+x^{-1 / 2}-5 / 2$ and notice that it has a minimum at $x^{*} \approx 2.31459$ where it reaches the value $f\left(x^{*}\right) \approx 0.110719$.

Define $x(\alpha)=\alpha\left(b-c_{\mathfrak{f}}\right) /\left(\alpha b-c_{\ell}\right)$. Notice that $x(\alpha)$ is strictly decreasing for $\alpha \geq 1$. Moreover, by Condition 1, $x(1)=\left(b-c_{\mathfrak{f}}\right) /\left(b-c_{\ell}\right) \geq 4, x(\alpha) \rightarrow\left(b-c_{\mathfrak{f}}\right) / b<1$ as $\alpha \rightarrow \infty$. This implies that there is only one intensity level of the affirmative action $\widehat{\alpha}>1$ such that $x(\widehat{\alpha})=x^{*} \approx 2.31459$. Moreover, since $f(x(\alpha)) \geq f(x(\widehat{\alpha}))$ for each $\alpha \in\left[1, \alpha^{e}\right]$, from equation (69) we have that

$$
\mathcal{A}(\alpha) \geq \frac{b-c_{\mathfrak{f}}}{2} f(x(\alpha)) \geq \frac{b-c_{\mathfrak{f}}}{2} f(x(\widehat{\alpha})) \approx 0.110719 \frac{b-c_{\mathfrak{f}}}{2}>0 .
$$

\section{A.4 Proof of Lemma 1}

Let us consider a given allocation rule $\varphi$, and a program with bias with intensity $\delta \in(0,1)$. Assume that $P^{*}=\left(p_{\ell}^{*}, p_{\mathfrak{f}}^{*}\right) \in S_{\ell} \times S_{\mathfrak{f}}$ is an undominated Nash equilibrium for such a program. Assume also that $S_{\ell}$ is $\delta$-consistent, and thus $\delta p_{\ell}^{*} \in S_{\ell}$. Then,

(a) for each $p_{\ell} \in S_{\ell}$,

$$
\pi_{\ell}^{\delta}\left(p_{\ell}^{*}, p_{\mathfrak{f}}^{*}\right)=\varphi_{\ell}\left(\delta p_{\ell}^{*}, p_{\mathfrak{f}}^{*}\right)\left(p_{\ell}^{*}-c_{\ell}\right) \geq \varphi_{\ell}\left(\delta p_{\ell}, p_{\mathfrak{f}}^{*}\right)\left(p_{\ell}-c_{\ell}\right)=\pi_{\ell}^{\delta}\left(p_{\ell}, p_{\mathfrak{f}}^{*}\right)
$$

and 
(b) for each $p_{\mathfrak{f}} \in S_{\mathfrak{f}}$,

$$
\pi_{\mathfrak{f}}^{\delta}\left(p_{\ell}^{*}, p_{\mathfrak{f}}^{*}\right)=\varphi_{\mathfrak{f}}\left(\delta p_{\ell}^{*}, p_{\mathfrak{f}}^{*}\right)\left(p_{\mathfrak{f}}^{*}-c_{\mathfrak{f}}\right) \geq \varphi_{\mathfrak{f}}\left(\delta p_{\ell}^{*}, p_{\mathfrak{f}}\right)\left(p_{\mathfrak{f}}-c_{\mathfrak{f}}\right)=\pi_{\mathfrak{f}}^{\delta}\left(p_{\ell}^{*}, p_{\mathfrak{f}}\right) .
$$

Let denote $\widehat{p}_{\ell}=\delta p_{\ell}^{*} \in S_{\ell}$. Observe that $\widehat{p}_{\ell}<b$. Then, equation 71 states that for each $p_{\mathfrak{f}} \in S_{\mathfrak{f}}$,

$$
\varphi_{\mathfrak{f}}\left(\widehat{p}_{\ell}, p_{\mathfrak{f}}^{*}\right)\left(p_{\mathfrak{f}}^{*}-c_{\mathfrak{f}}\right) \geq \varphi_{\mathfrak{f}}\left(\widehat{p}_{\ell}, p_{\mathfrak{f}}\right)\left(p_{\mathfrak{f}}-c_{\mathfrak{f}}\right) .
$$

Therefore, $p_{\mathfrak{f}}^{*}$ is a best-response for the foreign provider for the subsidy program with intensity $1 / \delta$ associated to the allocation rule $\varphi$ when the local provider selects the price $\widehat{p}_{\ell}$.

Assume that $\left(\widehat{p}_{\ell}, p_{\mathfrak{f}}^{*}\right)$ is not an undominated Nash equilibrium for the program with subsidy with intensity $\alpha=1 / \delta$. Then, by equation $(72)$, and since $\alpha \widehat{p}_{\ell}=p_{\ell}^{*} \leq b$, it should be the case that

$$
\pi_{\ell}^{\alpha C}\left(p_{\ell}^{\prime}, p_{\mathfrak{f}}^{*}\right)>\pi_{\ell}^{\alpha C}\left(\widehat{p}_{\ell}, p_{\mathfrak{f}}^{*}\right)=\varphi_{\ell}\left(\widehat{p}_{\ell}, p_{\mathfrak{f}}^{*}\right)\left(\alpha \widehat{p}_{\ell}-c_{\ell}\right)
$$

for some $p_{\ell}^{\prime} \in S_{\ell}$. Since the subsidy program satisfies the safeguard clause, and the local provider is selecting undominated prices, there is no loss of generality in assuming that $\alpha p_{\ell}^{\prime} \leq b$. Define $\bar{p}_{\ell}=\alpha p_{\ell}^{\prime}=p_{\ell}^{\prime} / \delta>p_{\ell}^{\prime}$. Then equation 73 can be rewritten as

$$
\varphi_{\ell}\left(\delta \bar{p}_{\ell}, p_{\mathfrak{f}}^{*}\right)\left(\bar{p}_{\ell}-c_{\ell}\right)>\varphi_{\ell}\left(\widehat{p}_{\ell}, p_{\mathfrak{f}}^{*}\right)\left(\alpha \widehat{p}_{\ell}-c_{\ell}\right)=\varphi_{\ell}\left(\delta p_{\ell}^{*}, p_{\mathfrak{f}}^{*}\right)\left(p_{\ell}^{*}-c_{\ell}\right) .
$$

Note that, since $\delta \bar{p}_{\ell}=p_{\ell}^{\prime} \leq b$, equation 74 contradicts that $\left(p_{\ell}^{*}, p_{\mathfrak{f}}^{*}\right)$ is an equilibrium for the program with bias with intensity $\delta$.

Now, assume that $\widehat{P}=\left(\widehat{p}_{\ell}, \widehat{p}_{\mathfrak{f}}\right)$ is an undominated Nash equilibrium for the subsidy program with intensity $\alpha$ associated to the allocation rule $\varphi$. Then,

(a) for each $p_{\ell} \in S_{\ell}$,

$$
\pi_{\ell}^{\alpha C}\left(\widehat{p}_{\ell}, \widehat{p}_{\mathfrak{f}}\right) \geq \pi_{\ell}^{\alpha C}\left(p_{\ell}, \widehat{p}_{\mathfrak{f}}\right)
$$

and

(b) for each $p_{\mathfrak{f}} \in S_{\mathfrak{f}}$,

$$
\pi_{\mathfrak{f}}^{\alpha C}\left(\widehat{p}_{\ell}, \widehat{p}_{\mathfrak{f}}\right)=\varphi_{\mathfrak{f}}\left(\widehat{p}_{\ell}, \widehat{p}_{\mathfrak{f}}\right)\left(\widehat{p}_{\mathfrak{f}}-c_{\mathfrak{f}}\right) \geq \varphi_{\mathfrak{f}}\left(\widehat{p}_{\ell}, p_{\mathfrak{f}}\right)\left(p_{\mathfrak{f}}-c_{\mathfrak{f}}\right)=\pi_{\mathfrak{f}}^{\alpha C}\left(\widehat{p}_{\ell}, p_{\mathfrak{f}}\right) .
$$

Denoting $p_{\ell}^{*}=\alpha \widehat{p}_{\ell}$ we have that equation $(76)$ states that, for each $p_{\mathfrak{f}} \in S_{\mathfrak{f}}$,

$$
\varphi_{\mathfrak{f}}\left(p_{\ell}^{*} / \alpha, \widehat{p}_{\mathfrak{f}}\right)\left(\widehat{p}_{\mathfrak{f}}-c_{\mathfrak{f}}\right) \geq \varphi_{\mathfrak{f}}\left(p_{\ell}^{*} / \alpha, p_{\mathfrak{f}}\right)\left(p_{\mathfrak{f}}-c_{\mathfrak{f}}\right) .
$$

Assume that $\left(p_{\ell}^{*}, \widehat{p}_{\mathfrak{f}}\right)$ is not an undominated Nash equilibrium for the program with bias with intensity $\delta=1 / \alpha$, associated to allocation rule $\varphi$. Note that, since $\ell$ selects undominated strategies when playing the subsidy program, $\widehat{p}_{\ell} \leq b / \alpha$, and thus $\delta p_{\ell}^{*} \leq b$. Then, by equation (77), there should be some $p_{\ell}^{\prime} \in S_{\ell}$ such that

$$
\varphi_{\ell}\left(\delta p_{\ell}^{\prime}, \widehat{p}_{\mathfrak{f}}\right)\left(p_{\ell}^{\prime}-c_{\ell}\right)>\varphi_{\ell}\left(\delta p_{\ell}^{*}, \widehat{p}_{\mathfrak{f}}\right)\left(p_{\ell}^{*}-c_{\ell}\right) .
$$


Denoting $\breve{p}_{\ell}=\delta p_{\ell}^{\prime}=p_{\ell}^{\prime} / \alpha$, equation $(78)$ becomes

$$
\varphi_{\ell}\left(\breve{p}_{\ell}, \widehat{p}_{\mathfrak{f}}\right)\left(\alpha \breve{p}_{\ell}-c_{\ell}\right)>\varphi_{\ell}\left(\widehat{p}_{\ell}, \widehat{p}_{\mathfrak{f}}\right)\left(\alpha \widehat{p}_{\ell}-c_{\ell}\right) \text {. }
$$

Note that, since $\delta<1$ and $p_{\ell}^{\prime} \leq b$, it follows that $\breve{p}_{\ell}=\delta p_{\ell}^{\prime}<b$, and thus equation 79 contradicts that $\left(\widehat{p}_{\ell}, \widehat{p}_{\mathfrak{f}}\right)$ is an undominated Nash equilibrium for the subsidy program with intensity $\alpha$ associated to the allocation rule $\varphi$.

\section{A.5 Equivalent Affirmative Action Programs in a Contest Setting}

In this appendix we clarify the relationship between our setting in the main text and contest games. We then prove an equivalence between affirmative action programs with bias and subsidy in contest games, similar to Lemma 1. This equivalence is well known in the contest literature, see e.g. Szidarovszky and Okuguchi (1997) and Esteban and Ray (1999). Similar to Example 4 in the main text, we show that this equivalence no longer holds when the strategy space of contestants is bounded (Che and Gale, 1997).

Contest games are usually formulated as forward auctions with an all-pay rule, while our setting in the main text postulates a reverse auction with a winner-pay rule. The following contest game is formulated as a forward auction and encompasses both a winner-pay and an all-pay rule. In addition, it differs from our setting in the main text in that we allow for any number of players.

Consider the following normal form game. The set of contestants is $I=\{1,2, \ldots, n\}$, where $n$ might be larger than two. The strategy space of each contestant is $S=\mathbb{R}_{+}$and we denote the effort of contestant $i$ by $e_{i}$. The vector of efforts is denoted by $E=\left(e_{1}, e_{2}, \ldots, e_{n}\right)$ and $E_{-i}$ indicates the same vector without the effort $e_{i}$. For each given contestant $i$, there is a cost function $k_{i}$ so that $k_{i}\left(e_{i}\right)$ denotes the $i$ 's cost when he exerted an effort of $e_{i}$. Associated to effort $e_{i}$ by contestant $i$ there is an effective effort $f_{i}\left(e_{i}\right)$. The allocation function $\varphi$ maps effective effort into win probabilities, that is, $\varphi(E \mid f) \in \mathbb{R}_{+}^{n}, \sum_{i}^{n} \varphi_{i}(E \mid f)=1$, and each entry of $\varphi$ is defined by $\varphi_{i}(E \mid f)=\varphi_{i}\left(f_{1}\left(e_{1}\right), f_{2}\left(e_{2}\right), \ldots, f_{n}\left(e_{n}\right)\right)$. Given the function $\varphi$, a valuation for winning $v_{i}$ and a parameter $\gamma \in[0,1]$, each contestant $i$ chooses effort to maximize his payoffs given by

$$
U_{i}(E \mid f)=\varphi_{i}(E \mid f)\left(v_{i}-\gamma k_{i}\left(e_{i}\right)\right)-(1-\gamma) k_{i}\left(e_{i}\right) .
$$

Notice that for $\gamma=0$ we have an all-pay contest, while for $\gamma=1$ the contest is winner-pay 52 We denote this normal form game by $\bar{\Gamma}=\{I, S, \varphi, U, f, k, \gamma\}$.

Two special cases of $\bar{\Gamma}$ are of interest. Denoting by $i d$ the identity function, define $\bar{\Gamma}^{f}=$ $\{I, S, \varphi, U, f, i d, \gamma\}$ and $\bar{\Gamma}^{k}=\{I, S, \varphi, U, i d, k, \gamma\}$. Notice that the special case of $\bar{\Gamma}^{f}$ in which $f_{i}\left(e_{i}\right)=\delta_{i} e_{i}$ constitutes the contest with bias $\delta=\left(\delta_{1}, \ldots, \delta_{i}, \ldots, \delta_{n}\right)$, while the special case of $\bar{\Gamma}^{k}$

\footnotetext{
${ }^{52}$ Note also that the payoffs in $(80)$ relate to the payoffs in $(1)$ in the main text as follows. Define $p_{i}=b-e_{i}$ and $c_{i}=b-v_{i}$. Using these expressions we see that both the FPA and the CPA defined in (2) and (3) respectively are non-decreasing on the effort $e_{i}$ of a contestant, as required in the contest setting. Moreover, the payoffs in (1) become the payoffs in 80 ) for $\gamma=1$.
} 
in which $k_{i}\left(e_{i}\right)=\alpha_{i} e_{i}$ defines the contest with subsidy $\alpha=\left(\alpha_{1}, \ldots, \alpha_{i}, \ldots, \alpha_{n}\right)$. Note also that these games define contests with a general bias in the sense that some contestants might benefit from the bias, while others might be harmed. An affirmative action program would set $\alpha_{i}<1$ and $\delta_{i}>1$ for some contestants (with $\alpha_{j}=\delta_{j}=1$ for the remaining agents), rather than $\alpha_{i}>1$ and $\delta_{i}<1$ as in the main text.

We have the following result which is related to (but different from) Lemma $1{ }^{53}$

Lemma 2 For each contestant $i$ let $k_{i}$ be the inverse of $f_{i}$, that is, $k_{i}\left(f_{i}\left(e_{i}\right)\right)=e_{i}$. Then $E^{*}=\left(e_{1}^{*}, \ldots, e_{n}^{*}\right)$ is an equilibrium for the game $\bar{\Gamma}^{f}$ if and only if the vector of efforts $\left(f_{1}\left(e_{1}^{*}\right), \ldots, f_{n}\left(e_{n}^{*}\right)\right)$ is an equilibrium for the game $\bar{\Gamma}^{k}$.

To prove Lemma 2 consider $\bar{\Gamma}^{f}$ and assume that $E^{*}=\left(e_{1}^{*}, e_{2}^{*}, \ldots, e_{n}^{*}\right)$ is an equilibrium for this game. This implies that for each contestant $i$ and effort level $e_{i}$ we have that

$$
\begin{aligned}
U_{i}\left(E^{*} \mid f\right) & =\varphi_{i}\left(f_{1}\left(e_{1}^{*}\right), \ldots f_{i}\left(e_{i}^{*}\right), \ldots, f_{n}\left(e_{n}^{*}\right)\right)\left(v_{i}-\gamma e_{i}^{*}\right)-(1-\gamma) e_{i}^{*} \geq \\
& \geq \varphi_{i}\left(f_{1}\left(e_{1}^{*}\right), \ldots f_{i}\left(e_{i}\right), \ldots, f_{n}\left(e_{n}^{*}\right)\right)\left(v_{i}-\gamma e_{i}\right)-(1-\gamma) e_{i}=U_{i}\left(e_{i}, E_{-i}^{*} \mid f\right)
\end{aligned}
$$

For each contestant $i$ let $\widehat{e}_{i}=f_{i}\left(e_{i}^{*}\right)$ and $e_{i}^{\prime}=f_{i}\left(e_{i}\right)$ for $e_{i} \neq e_{i}^{*}$. Using this notation and the fact that $k_{i}$ is the inverse of $f_{i}$ the inequality in (81) is equivalent to

$$
\begin{aligned}
& \varphi_{i}\left(\widehat{e}_{1}, \ldots \widehat{e}_{i}, \ldots, \widehat{e}_{n}\right)\left(v_{i}-\gamma k_{i}\left(\widehat{e}_{i}\right)\right)-(1-\gamma) k_{i}\left(\widehat{e}_{i}\right) \geq \\
\geq & \varphi_{i}\left(\widehat{e}_{1}, \ldots e_{i}^{\prime}, \ldots, \widehat{e}_{n}\right)\left(v_{i}-\gamma k_{i}\left(e_{i}^{\prime}\right)\right)-(1-\gamma) k_{i}\left(e_{i}^{\prime}\right),
\end{aligned}
$$

for all $e_{i}^{\prime}$. Hence, we have that $U_{i}(\widehat{E} \mid f) \geq U_{i}\left(e_{i}^{\prime}, \widehat{E}_{-i} \mid f\right)$ for all $e_{i}^{\prime}$. Therefore, for each contestant $i$ the effort level $\widehat{e}_{i}$ is a best-response to $\widehat{E}_{-i}$ in the game $\bar{\Gamma}^{k}$, and thus $\widehat{E}$ is an equilibrium for $\bar{\Gamma}^{k}$.

In contrast to Lemma 2, Lemma 1 assumes the safeguard clause. The reason is that in the model of the main text there is a bound on the strategy space. Che and Gale (1997) introduce budget constraints in contest models. The next example shows that when contestants face budget constraints additional assumptions are needed.

Example 7 Consider $\bar{\Gamma}^{f}$. Let $n=2, v_{1}=v_{2}=v, \gamma=1$ and consider the so-called Tullock contest. That is, the win probability of a contestant is given by $\varphi_{i}^{T}(E \mid f)=f_{i}\left(e_{i}\right) /\left(f_{1}\left(e_{1}\right)+\right.$ $\left.\left.f_{2}\left(e_{2}\right)\right)\right)$ when at least one $f_{i}\left(e_{i}\right)>0$ and $1 / 2$ otherwise. Let $f_{i}\left(e_{i}\right)=4 e_{i}$ for both contestants. Standard derivations show that in equilibrium $e_{1}^{*}=e_{2}^{*}=v / 4$.

\footnotetext{
${ }^{53}$ Lemma 1 and the next lemma are different, because the program with subsidy in the main text and in the contest setting coincide only when $\alpha_{i}=1$ for all players. To see this consider the game $\bar{\Gamma}$. Let $\gamma=1$ so that the payoffs in (1) become the payoffs in (80) when (as in footnote 52) $p_{i}=b-e_{i}$ and $c_{i}=b-v_{i}$. Now compare the payoffs in $\bar{\Gamma}^{\alpha}$ to those with the program with subsidy $\alpha$ in the main text. In the former case we have $\varphi_{i}(P)\left(\alpha p_{i}+(1-\alpha) b-c_{i}\right)$, while under the latter (6) stipulates that $\varphi_{i}(P)\left(\alpha p_{i}-c_{i}\right)$. The fact that the two programs with subsidy $\alpha$ are different implies that Lemmata 1 and 2 are independent rather than that one is more general than the other.
} 
Now consider $\bar{\Gamma}^{k}$. Let $k_{i}\left(e_{i}\right)=e_{i} / 4$ for both contestants. Again, standard derivations show that in equilibrium $\widehat{e}_{1}=\widehat{e}_{2}=v$. This is, of course, consistent with Lemma 2 , as $\widehat{e}_{i}=4 e_{i}^{*}$ for both contestants.

Suppose now that contestants face budget constraints $w_{i}=v / 2$. These constraints are not binding in the first game and the equilibrium is still $e_{1}^{*}=e_{2}^{*}=v / 4$. In the second game, however, $\widehat{e}_{1}=\widehat{e}_{2}=v$ is no longer feasible and Che and Gale (1997) have shown that in equilibrium the contestants' effort equals their wealth level $w_{i}=v / 2$. Consequently, when contestants face budget constraints, Lemma 2 does not hold. 